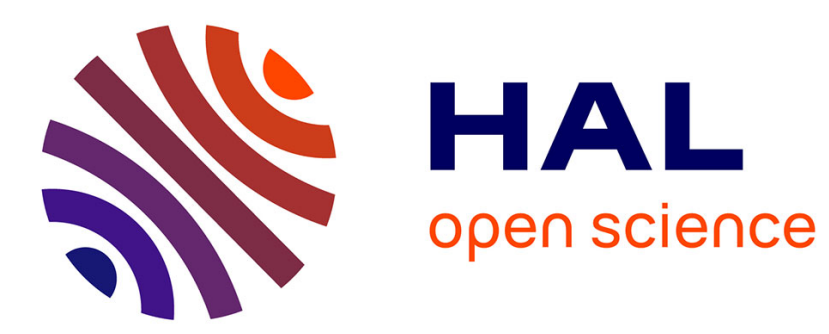

\title{
Stochastic expansion for the pricing of call options with discrete dividends
}

Pierre Etoré, Emmanuel Gobet

\section{To cite this version:}

Pierre Etoré, Emmanuel Gobet. Stochastic expansion for the pricing of call options with discrete dividends. Applied Mathematical Finance, 2012, 19 (3), pp.233-264. 10.1080/1350486X.2011.620397. hal-00507787

\section{HAL Id: hal-00507787 \\ https://hal.science/hal-00507787}

Submitted on 31 Jul 2010

HAL is a multi-disciplinary open access archive for the deposit and dissemination of scientific research documents, whether they are published or not. The documents may come from teaching and research institutions in France or abroad, or from public or private research centers.
L'archive ouverte pluridisciplinaire HAL, est destinée au dépôt et à la diffusion de documents scientifiques de niveau recherche, publiés ou non, émanant des établissements d'enseignement et de recherche français ou étrangers, des laboratoires publics ou privés. 


\title{
Stochastic expansion for the pricing of call options with discrete dividends*
}

\author{
P. Étoré and E. Gobet \\ Grenoble University and CNRS \\ Laboratoire Jean Kuntzmann \\ 51 rue des Mathématiques \\ F - 38041 Grenoble cedex 9 \\ pierre.etore@imag.fr, emmanuel.gobet@imag.fr
}

July 31, 2010

\begin{abstract}
In the context of an asset paying affine-type discrete dividends, we present closed analytical approximations for the pricing of European vanilla options in the Black-Scholes model with time-dependent parameters. They are obtained using a stochastic Taylor expansion around a shifted lognormal proxy model. The final formulae are respectively first, second and third order approximations w.r.t. the fixed part of the dividends. Using Cameron-Martin transformations, we provide explicit representations of the correction terms as Greeks in the Black-Scholes model. The use of Malliavin calculus enables us to provide tight error estimates for our approximations. Numerical experiments show that the current approach yields very accurate results, in particular compared to known approximations of [BGS03, VW09], and quicker than the iterated integration procedure of [HHL03] or than the binomial tree method of [VN06].
\end{abstract}

${ }^{*}$ This research was partially supported by University Joseph Fourier (MSTIC grant REFINE) and by Fondation du Risque - Chaire Risques Financiers Société Générale Ecole Polytechnique - ENPC (second author). 
Keywords: equity option, discrete dividend, stochastic approximation, analytic formula.

Classification. MSC (2000): 41XX - 60HXX. JEL Classification: G13.

\section{Introduction}

Usually, stocks pay dividends, which modelling is a non-trivial issue. This has also some implications regarding the computational point of view, that is to efficiently price vanilla options written on the stock and to quickly calibrate the stock model. If we use a deterministic continuously paid dividend yield and we assume that the asset dynamics is lognormal, then we can extend the classical pricing Black-Scholes formula by minor modifications (see equation (1.6)). Assuming continuous dividends is an approximation that can be justified if one considers a large portfolio of stocks paying individually discrete dividends. However, for a single stock, considering discrete dividends is more realistic and this is our framework. Actually, our aim is to provide efficient approximation formulae for Call options written on a single asset paying discrete dividends. For this, we follow an approach based on stochastic expansions, using stochastic analysis tools, approach that has been similarly developed in a series of papers [BGM09, BGM10a, BGM10c, BGM10b].

In the literature, several works handle the issues of numerical computation of the call price when dividends are discrete. Of course, a Monte Carlo approach is still possible, whatever the asset model and the dividend model are, but usually it is not competitive comparing to analytical approximations or one-dimensional tree methods. Several works [HHL03, VN06, VW09] rely on the dynamic programming equation between two successive dividend dates, say $t_{i}$ and $t_{i+1}$. Namely, denote by $C(t, S)$ the option price function at time $t$ for an asset equal to $S$, write $d_{i}(S)$ for the (known) dividend policy modelling the dividend paid at time $t_{i}$ (it depends on the asset): then, for a Markovian price process $\left(S_{t}\right)_{0 \leq t \leq T}$ and deterministic interest rates $\left(r_{t}\right)_{0 \leq t \leq T}$ we have

$$
C\left(t_{i}, S_{t_{i}}\right)=\mathbb{E}\left(e^{-\int_{t_{i}}^{t_{i+1}} r_{s} d s} C\left(t_{i+1}, S_{t_{i+1}^{-}}-d_{i+1}\left(S_{t_{i+1}^{-}}\right)\right) \mid S_{t_{i}}\right),
$$

the expectation being computed under the risk-neutral pricing measure. In [HHL03, the authors discuss in details the proper choice of dividend policy. In addition, they compute the price function $C(.,$.$) using integration methods$ 
to compute the expectation in (0.1), for tractable dynamics of $S$ (lognormal for instance). This numerical approach is exact (up to integration error) but it is computationally intensive. In [VN06], for a piecewise lognormal asset, the authors design a binomial tree method to solve (0.1). The main difficulty in using a tree method is the a priori non-recombination of the nodes at the dividend dates. The authors overcome this problem by using interpolation techniques between nodes. They also prove the convergence of their approximation, as the number of steps in the tree method goes to infinity. Finally, in [VW09, still for a piecewise lognormal model and for a fixed dividend policy $d_{i+1}(S)=\delta_{i+1}$, the authors expand the equality 0.1$)$ w.r.t. $\left(\delta_{i}\right)_{i}$ and provide an approximation formula involving the Black-Scholes formula and its Greeks w.r.t. the spot. For $n$ dividend dates, the number of BS price/greeks to compute grows exponentially like $3^{n}$, as the number of dividend dates increase (in their tests, the authors take $n=7$, giving 2187 terms to evaluate); it may be very costly. Another approach is developed by Bos, Gairat and Shepeleva in [BGS03]: they give an approximative formula for the equivalent implied Black-Scholes volatility, in order to take into account the dividends. It is obtained by a suitable average of the instantaneous volatility of the asset paying dividends.

In this work, we derive an alternative expansion of the price function w.r.t. the dividends. The resulting approximation also writes as a combination of BS formulae and Greeks w.r.t. the strike (and not the spot). Compared to [VW09], our second order approximation formula requires the evaluation of only $45 \mathrm{BS}$ price/greeks for 7 dividend dates. Thus, at least regarding the computational cost, it improves [VW09] and it is similar to [BGS03]. Moreover, our assumption on the dividend policy is less restrictive, see below. In addition, the numerical results show an excellent accuracy of our formulae.

In the current work, the model for $S$ is a piecewise lognormal model (with time-dependent parameters) and the dividend policy is affine in $S$, i.e. including a fixed and a proportional part:

$$
d_{i}(S)=\delta_{i}+y_{i} S
$$

One drawback of this model is that after a dividend payment, the asset price may become negative because the relation $d_{i}(S) \leq S$ may be violated for small $S$. However, in most of our numerical tests, the probability of such event is very small (see Tables 5 and 6); presumably, it has a very small impact on the call price. Although this model of dividend policy is quite 
simple, it is often used by practitioners. In future research, we intend to improve it, by leveraging the works by [KR05, Bue10].

To obtain our approximations, we choose a model proxy obtained by averaging the future dividends. Then we use stochastic expansion techniques in the spirit of the work [BGM09, BGM10a, BGM10c, BGM10b]. A significative part of effort is made to derive non asymptotic error estimates, justifying the first order, second or third order approximation. This approach is quite flexible and we believe that this work paves the way for future research in order to obtain analytical approximations of call price with discrete dividends including Heston or local volatilities, or stochastic interest rate as well.

The organization of the paper is the following. In the next section, we define the model and notations used throughout the work. In Section 2, we state our main approximation results about first, second and third order approximation formulae for the call price. Extensions to the computation of the Delta are given as well. Section 3 is devoted to the proof of technical results involving Malliavin calculus. Numerical tests are presented in Section 4 .

\section{Model and notations}

\subsection{Financial framework}

We consider a standard complete financial market, with a traded risky asset on which an European vanilla option with maturity $T$ is written. In our study, specifically the asset pays dividends at known dates $0<t_{1}<\ldots<t_{n} \leq T<$ $+\infty(n \geq 1)$. We assume that the second date $t_{2}$ (whenever existing when $n>1)$ is larger than one year $\left(t_{2} \geq 1\right)$ : this is not a practical restriction since usually dividends are paid once a year. At time $t_{i}$, the amount of dividends is splitted into a proportional part $y_{i} \in\left[0,1\left[\right.\right.$ and a fixed part $\delta_{i} \geq 0$. To make clear the asset dependency w.r.t. the dividends, we denote by $\left(S_{t}^{(y, \delta)}\right)_{t}$ the asset price process. Then, the amount of dividend at time $t_{i}$ equals to

$$
\delta_{i}+y_{i} S_{t_{i}-}^{(y, \delta)}
$$

which implies that the asset price jumps downwards to

$$
S_{t_{i}}^{(y, \delta)}=S_{t_{i}-}^{(y, \delta)}-\left[\delta_{i}+y_{i} S_{t_{i}-}^{(y, \delta)}\right]=S_{t_{i}-}^{(y, \delta)}\left(1-y_{i}\right)-\delta_{i}
$$

just after the dividend payment. 
Moreover, we assume that between two dividend dates, the asset follows an Ito dynamics with a time-dependent volatility $\left(\sigma_{t}\right)_{t}$. Since we focus only pricing/hedging issues, we write the dynamics of $S^{(y, \delta)}$ under the (unique) risk-neutral measure $\mathbb{Q}$ : between two dividend dates it writes

$$
d S_{t}^{(y, \delta)}=\sigma_{t} S_{t}^{(y, \delta)} d W_{t}+\left(r_{t}-q_{t}\right) S_{t}^{(y, \delta)} d t
$$

where $W$ is a standard $\mathbb{Q}$-Brownian motion. In the above equation, $\left(q_{t}\right)_{t}$ should be interpreted as a (deterministic) repo rate. The interest rate $\left(r_{t}\right)_{t}$ is assumed to be deterministic. The functions $\left(r_{t}\right)_{t}$ and $\left(q_{t}\right)_{t}$ are bounded.

\subsection{Assumptions and notations}

Assumptions. It is not a practical restriction to assume that the ratio between fixed dividends and the current asset $S_{0}$ remains bounded by a constant $c_{\delta}$ (likely smaller than 1 in practice): $\sup _{i} \delta_{i} / S_{0} \leq c_{\delta}$.

- In addition, for some of our results we may assume that for the first dividend date, the ratio is small enough in the sense

$$
\frac{\delta_{1}}{S_{0}\left(1-y_{1}\right)}<1
$$

- For some results, we impose a non-degenaracy condition on the model (ellipticity condition):

$$
\forall t \in[0, T], \quad 0<\underline{\sigma} \leq \sigma_{t} \leq \bar{\sigma}
$$

Notations. For convenience, we repeatedly use the following notations.

- We write $D_{t}$ for the discount factor: $D_{t}=\exp \left(-\int_{0}^{t}\left(r_{s}-q_{s}\right) d s\right)$.

- We write $M_{t}=\exp \left(\int_{0}^{t} \sigma_{s} d W_{s}-\frac{1}{2} \int_{0}^{t} \sigma_{s}^{2} d s\right)$ for the log-normal martingale with volatility $\left(\sigma_{s}\right)_{0 \leq s \leq T}$.

- We write $S$ for the (fictitious) asset without dividends:

$$
d S_{t}=\sigma_{t} S_{t} d W_{t}+\left(r_{t}-q_{t}\right) S_{t} d t,
$$

and its initial value is $S_{0}=S_{0}^{(y, \delta)}$. Thus,

$$
S_{t}=S_{0} \frac{M_{t}}{D_{t}} .
$$


- We set $\pi_{i, n}:=\prod_{j=i+1}^{n}\left(1-y_{j}\right)=\left(1-y_{n}\right) \cdots\left(1-y_{i+1}\right)$ for $0 \leq i \leq n$ with the convention that $\prod_{j=n+1}^{n}\left(1-y_{j}\right)=1$ (so that $\left.\pi_{n, n}=1\right)$.

- For the sake of conciseness, we may use the simplified notation

$$
\hat{\delta}_{i}=\delta_{i} \pi_{i, n} \frac{D_{t_{i}}}{D_{T}}
$$

For a given strike $K>0$, the shifted strike $K^{(y, \delta)}$ will play an important role in our approximation formulae:

$$
K^{(y, \delta)}=K+\sum_{i=1}^{n} \delta_{i} \pi_{i, n} \frac{D_{t_{i}}}{D_{T}}=K+\sum_{i=1}^{n} \hat{\delta}_{i}
$$

- We write $A \leq{ }_{c} B$ when $A \leq c B$ for some constant $c$ which depends smoothly on the model parameters. The constant $c$ remains bounded as the maturity $T$ or the parameters $r, q, \sigma, y, c_{\delta}$ go to 0 . The constant $c$ may depend on the ratio $\bar{\sigma} / \underline{\sigma} \geq 1$ and on the number $n$ of dividend dates. The dependency w.r.t. $S_{0}$ is systematically written. When relevant, explicit dependencies w.r.t. parameters are indicated.

- In the error analysis, we use repeatedly the standard estimates $\mathbb{E}\left(\sup _{s \leq T} S_{s}^{k}\right) \leq_{c}$ $S_{0}^{k}$ for any $k \in \mathbb{R}$.

\subsection{Preliminary relations}

With the previous notations, we easily deduce

$$
S_{t}^{(y, \delta)}= \begin{cases}S_{t} & \text { if } t<t_{1} \\ S_{t_{i}}^{(y, \delta)} \frac{S_{t}}{S_{t_{i}}}=\left(1-y_{i}\right) S_{t_{i}-}^{(y, \delta)} \frac{S_{t}}{S_{t_{i}}}-\delta_{i} \frac{S_{t}}{S_{t_{i}}} & \text { if } t_{i} \leq t<t_{i+1} \text { for } i<n \\ & \text { or } t_{i} \leq t \leq T \text { for } i=n .\end{cases}
$$

Then, an easy induction (detailed in Appendix) leads to the following

Lemma 1.1. We have $S_{T}^{(y, \delta)}=\pi_{0, n} S_{T}-\sum_{i=1}^{n} \delta_{i} \pi_{i, n} \frac{S_{T}}{S_{t_{i}}}$. 
A case of special interest corresponds to proportional dividends only ( $\delta_{i} \equiv$ 0 ) for which we have $S_{T}^{(y, 0)}:=\pi_{0, n} S_{T}$. This is a lognormal random variable and an explicit formula for the related call price is available via the BlackScholes formula:

$$
\mathbb{E}\left(e^{-\int_{0}^{T} r_{s} d s}\left(S_{T}^{(y, 0)}-K\right)_{+}\right)=\operatorname{Call}^{B S}\left(\pi_{0, n} S_{0}, K\right)
$$

with

$$
\begin{gathered}
\operatorname{Call}^{B S}(x, k)=x e^{-\int_{0}^{T} q_{s} d s} \mathcal{N}\left[d_{+}(x, k)\right]-k e^{-\int_{0}^{T} r_{s} d s} \mathcal{N}\left[d_{-}(x, k)\right] \\
d_{ \pm}(x, k)=\frac{1}{\sqrt{\int_{0}^{T} \sigma_{s}^{2} d s}} \log \left(\frac{x}{k}\right)+\frac{1}{\sqrt{\int_{0}^{T} \sigma_{s}^{2} d s}} \int_{0}^{T}\left(r_{s}-q_{s} \pm \frac{1}{2} \sigma_{s}^{2}\right) d s
\end{gathered}
$$

$\mathcal{N}$ being the cumulative distribution function of a standard Gaussian variable. Note that the price depends of course of $\left(r_{t}\right)_{t},\left(q_{t}\right)_{t}$ and $\left(\sigma_{t}\right)_{t}$, but we choose in our notations to highlight the dependency w.r.t. the initial value and the strike. Indeed this plays a crucial role in our calculations.

The case $\delta_{i} \equiv 0$ is important for our study since it serves to find a proxy for the case with fixed dividends. The proxy will not be directly given by the model with $\delta \equiv 0$, but by this model shifted by the expectation of the fixed dividends. In other words, in view of Lemma 1.1, the proxy is defined by

$$
\bar{S}_{T}^{(y, \delta)}:=\pi_{0, n} S_{T}-\mathbb{E}\left(\sum_{i=1}^{n} \delta_{i} \pi_{i, n} \frac{S_{T}}{S_{t_{i}}}\right)=\pi_{0, n} S_{T}-\sum_{i=1}^{n} \delta_{i} \pi_{i, n} \frac{D_{t_{i}}}{D_{T}}=\pi_{0, n} S_{T}-\sum_{i=1}^{n} \hat{\delta}_{i},
$$

recalling the definition $\hat{\delta}_{i}=\delta_{i} \pi_{i, n} \frac{D_{t_{i}}}{D_{T}}$. This is a shifted lognormal random variable, thus the computation of $\mathbb{E}\left(e^{-\int_{0}^{T} r_{s} d s}\left(\bar{S}_{T}^{(y, \delta)}-K\right)_{+}\right)$is still explicit, by taking the shifted strike variable $K^{(y, \delta)}$ (defined in (1.4)) in the BlackScholes formula:

$$
\mathbb{E}\left(e^{-\int_{0}^{T} r_{s} d s}\left(\bar{S}_{T}^{(y, \delta)}-K\right)_{+}\right)=\operatorname{Call}^{B S}\left(\pi_{0, n} S_{0}, K^{(y, \delta)}\right) .
$$

The above quantity stands for the main term of our expansion formula of $\mathbb{E}\left(e^{-\int_{0}^{T} r_{s} d s}\left(S_{T}^{(y, \delta)}-K\right)_{+}\right)$(see Theorems 2.3 and 2.4). The asymptotics underlying the expansion is $\sup _{i} \delta_{i} / S_{0} \rightarrow 0$ (small fixed dividends).

Our next purpose is now twofold: first, to provide correction terms, that will enable us to achieve a remarkable accuracy. Second, to give tight error estimates w.r.t. the model parameters. 


\section{Main results}

Our analysis is based on Taylor expansions and smart computations of the correction terms using the proxy $\bar{S}_{T}^{(y, \delta)}$. In order to study the distance to the proxy, we use Lemma 1.1 and equality (1.7) to write

$$
\begin{aligned}
S_{T}^{(y, \delta)} & =S_{T}^{(y, 0)}-\sum_{i=1}^{n} \delta_{i} \pi_{i, n} \frac{D_{t_{i}}}{D_{T}}\left(1+\frac{M_{T}}{M_{t_{i}}}-1\right) \\
& =\bar{S}_{T}^{(y, \delta)}-\sum_{i=1}^{n} \hat{\delta}_{i}\left(\frac{M_{T}}{M_{t_{i}}}-1\right) .
\end{aligned}
$$

Our ultime purpose is to approximate $\mathbb{E}\left(e^{-\int_{0}^{T} r_{s} d s} h\left(S_{T}^{(y, \delta)}-K\right)\right)$ for $h(x)=x_{+}$ (that is the Call price). Actually, the derivation of the approximation and the error estimation are simpler when the function $h$ is smoother than for Call/Put option. We start by this case in subsection 2.1, for the convenience of the reader. Then, handling call payoffs $h(x)=x_{+}$requires more technicalities related to Malliavin calculus and we tackle this case later in subsection 2.2 and section 3 .

\subsection{Taylor expansion for smooth $h$}

The degree $k(\geq 1)$ of smoothness of $h$ is defined as follows:

$\left(\mathbf{H}_{k}\right)$ The function $h($.$) is (k-1)$-times continuously differentiable and the $(k-1)$-th derivative is almost everywhere differentiable. Moreover, the derivatives are polynomially bounded: for some positive constants $C$ and $p$ one has $|h(x)|+\sum_{j=1}^{k}\left|\partial_{x}^{j} h(x)\right| \leq C\left(1+|x|^{p}\right)$ for any $x \in \mathbb{R}$.

First order approximation. We aim at approximating $\mathbb{E}\left(e^{-\int_{0}^{T} r_{s} d s} h\left(S_{T}^{(y, \delta)}\right.\right.$ $K)$ ) for functions $h$ satisfying $\left(\mathbf{H}_{2}\right)$. Using a first order Taylor expansion we have

$$
\begin{aligned}
& \mathbb{E}\left[e^{-\int_{0}^{T} r_{s} d s} h\left(S_{T}^{(y, \delta)}-K\right)\right]=\mathbb{E}\left[e^{-\int_{0}^{T} r_{s} d s} h\left(\bar{S}_{T}^{(y, \delta)}-K\right)\right] \\
& -\sum_{i=1}^{n} \hat{\delta}_{i} \mathbb{E}\left[e^{-\int_{0}^{T} r_{s} d s} h^{\prime}\left(\bar{S}_{T}^{(y, \delta)}-K\right)\left(\frac{M_{T}}{M_{t_{i}}}-1\right)\right]+\operatorname{Error}_{2}(h)
\end{aligned}
$$

where $\left|\operatorname{Error}_{2}(h)\right| \leq_{c}\left(1+S_{0}^{p}\right) \sup _{i}\left(\delta_{i}\left\|\frac{M_{T}}{M_{t_{i}}}-1\right\|_{3}\right)^{2}$. By standard computations (see also Lemma 3.3), we have

$$
\left\|\frac{M_{T}}{M_{t_{i}}}-1\right\|_{p} \leq_{c_{p}} \bar{\sigma} \sqrt{T-t_{i}}
$$


for any $p \geq 1$. It readily follows that $\left|\operatorname{Error}_{2}(h)\right| \leq_{c}\left(1+S_{0}^{p}\right) \sup _{i}\left(\delta_{i} \bar{\sigma} \sqrt{T-t_{i}}\right)^{2}$.

It remains to simplify the terms in the summation of $(2.2)$. For each $1 \leq i \leq n$ we write

$$
\begin{aligned}
\mathbb{E}\left[e^{-\int_{0}^{T} r_{s} d s} h^{\prime}\left(\bar{S}_{T}^{(y, \delta)}-K\right)\left(\frac{M_{T}}{M_{t_{i}}}-1\right)\right]= & \mathbb{E}\left[e^{-\int_{0}^{T} r_{s} d s} h^{\prime}\left(\bar{S}_{T}^{(y, \delta)}-K\right) \frac{M_{T}}{M_{t_{i}}}\right] \\
& -\mathbb{E}\left[e^{-\int_{0}^{T} r_{s} d s} h^{\prime}\left(\bar{S}_{T}^{(y, \delta)}-K\right)\right] .
\end{aligned}
$$

The second term on the right hand side can be rewritten using a derivative w.r.t. $K$ (the assumptions on $h$ allow us to interchange derivation and expectation):

$$
\begin{aligned}
\mathbb{E}\left[e^{-\int_{0}^{T} r_{s} d s} h^{\prime}\left(\bar{S}_{T}^{(y, \delta)}-K\right)\right] & =-\partial_{K} \mathbb{E}\left[e^{-\int_{0}^{T} r_{s} d s} h\left(\bar{S}_{T}^{(y, \delta)}-K\right)\right] \\
& =-\left.\partial_{k} \mathbb{E}\left[e^{-\int_{0}^{T} r_{s} d s} h\left(\pi_{0, n} S_{T}-k\right)\right]\right|_{k=K^{(y, \delta)}}
\end{aligned}
$$

This representation is useful for the call/put case to interpret expansion terms as Greeks (and thus explicit terms).

Note that we have in general, for any multiplicative constant $\alpha>0$, any strike $k$, and any derivative of order $m \in \mathbb{N}$ of any sufficiently smooth function $h$,

$$
\mathbb{E}\left[e^{-\int_{0}^{T} r_{s} d s} h^{(m)}\left(\alpha S_{T}-k\right)\right]=(-1)^{m} \partial_{k}^{m} \mathbb{E}\left[e^{-\int_{0}^{T} r_{s} d s} h\left(\alpha S_{T}-k\right)\right] .
$$

Regarding to the first term in the r.h.s. of (2.4), we interpret the factor $\frac{M_{T}}{M_{t_{i}}}$ as a change of measure on $\mathcal{F}_{T}$. Under the new induced measure $\mathbb{Q}^{i}$, $\bar{W}_{t}=W_{t}-\int_{0}^{t} \sigma_{s} \mathbf{1}_{t_{i} \leq s \leq T} d s$ is a Brownian motion. Then, $S_{T}$ under $\mathbb{Q}^{i}$ has the same law as $S_{T} e^{\int_{t_{i}}^{T} \sigma_{s}^{2} d s}$ under $\mathbb{Q}$. Thus,

$$
\begin{aligned}
& \mathbb{E}\left[e^{-\int_{0}^{T} r_{s} d s} h^{\prime}\left(\bar{S}_{T}^{(y, \delta)}-K\right) \frac{M_{T}}{M_{t_{i}}}\right] \\
= & \mathbb{E}\left[e^{-\int_{0}^{T} r_{s} d s} h^{\prime}\left(\pi_{0, n} e^{\int_{t_{i}}^{T} \sigma_{s}^{2} d s} S_{T}-\sum_{i=1}^{n} \hat{\delta}_{i}-K\right)\right] \\
= & -\left.\partial_{k} \mathbb{E}\left[e^{-\int_{0}^{T} r_{s} d s} h\left(\pi_{0, n} e^{\int_{t_{i}}^{T} \sigma_{s}^{2} d s} S_{T}-k\right)\right]\right|_{k=K^{(y, \delta)}}
\end{aligned}
$$

using (2.6) at the last line. Combining the above equality with 2.5 and (2.4), and plugging this into (2.2), we obtain our first main result. 
Theorem 2.1. For a smooth function $h$ satisfying $\left(\mathbf{H}_{2}\right)$, we have

$$
\begin{aligned}
& \mathbb{E}\left[e^{-\int_{0}^{T} r_{s} d s} h\left(S_{T}^{(y, \delta)}-K\right)\right] \\
= & \mathbb{E}\left[e^{-\int_{0}^{T} r_{s} d s} h\left(\pi_{0, n} S_{T}-K^{(y, \delta)}\right)\right] \\
& +\sum_{i=1}^{n} \hat{\delta}_{i}\left(\left.\partial_{k} \mathbb{E}\left[e^{-\int_{0}^{T} r_{s} d s} h\left(\pi_{0, n} e^{\int_{t_{i}}^{T} \sigma_{s}^{2}} S_{T}-k\right)\right]\right|_{k=K^{(y, \delta)}}\right. \\
& \left.\quad-\left.\partial_{k} \mathbb{E}\left[e^{-\int_{0}^{T} r_{s} d s} h\left(\pi_{0, n} S_{T}-k\right)\right]\right|_{k=K^{(y, \delta)}}\right)+\operatorname{Error}_{2}(h),
\end{aligned}
$$

with $\left|\operatorname{Error}_{2}(h)\right| \leq c\left(1+S_{0}^{p}\right) \sup _{i}\left(\delta_{i} \bar{\sigma} \sqrt{T-t_{i}}\right)^{2}$.

Note that in the terms on the r.h.s. of the above equality, the function $h$ is systematically evaluated at a shifted lognormal random variable. This allows for simple and tractable one-dimensional numerical computations.

This approximation formula is a first-order expansion formula w.r.t. the fixed dividends since the error is a $O\left(\sup _{i} \delta_{i}^{2}\right)$.

Second order approximation. Applying the same kind of arguments, we can derive another formula, which residual terms are of order three w.r.t. the fixed dividends.

Theorem 2.2. For a smooth function $h$ satisfying $\left(\mathbf{H}_{3}\right)$, we have

$$
\begin{aligned}
& \mathbb{E}\left[e^{-\int_{0}^{T} r_{s} d s} h\left(S_{T}^{(y, \delta)}-K\right)\right] \\
= & \mathbb{E}\left[e^{-\int_{0}^{T} r_{s} d s} h\left(\pi_{0, n} S_{T}-K^{(y, \delta)}\right)\right] \\
& +\sum_{i=1}^{n} \hat{\delta}_{i}\left(\left.\partial_{k} \mathbb{E}\left[e^{-\int_{0}^{T} r_{s} d s} h\left(\pi_{0, n} e^{\int_{t_{i}}^{T} \sigma_{s}^{2}} S_{T}-k\right)\right]\right|_{k=K}(y, \delta)\right. \\
& \left.-\left.\partial_{k} \mathbb{E}\left[e^{-\int_{0}^{T} r_{s} d s} h\left(\pi_{0, n} S_{T}-k\right)\right]\right|_{k=K^{(y, \delta)}}\right) \\
+ & \frac{1}{2}\left(\left.\sum_{1 \leq i, j \leq n} \hat{\delta}_{i} \hat{\delta}_{j} \partial_{k}^{2} \mathbb{E}\left[e^{-\int_{0}^{T} r_{s} d s} h\left(\pi_{0, n} e^{\int_{t_{i}}^{T} \sigma_{s}^{2} d s+\int_{t_{j}}^{T} \sigma_{s}^{2} d s} S_{T}-k\right)\right]\right|_{k=K^{(y, \delta)}} e^{\int_{t_{i} \vee t_{j}}^{T} \sigma_{s}^{2} d s}\right. \\
& \quad-\left.2\left(\sum_{j=1}^{n} \hat{\delta}_{j}\right) \sum_{i=1}^{n} \hat{\delta}_{i} \partial_{k}^{2} \mathbb{E}\left[e^{-\int_{0}^{T} r_{s} d s} h\left(\pi_{0, n} e^{\int_{t_{i}}^{T} \sigma_{s}^{2} d s} S_{T}-k\right)\right]\right|_{k=K^{(y, \delta)}} \\
& \left.+\left.\left(\sum_{j=1}^{n} \hat{\delta}_{j}\right)^{2} \partial_{k}^{2} \mathbb{E}\left[e^{-\int_{0}^{T} r_{s} d s} h\left(\pi_{0, n} S_{T}-k\right)\right]\right|_{k=K^{(y, \delta)}}\right)+\operatorname{Error}_{3}(h),
\end{aligned}
$$

with $\left|\operatorname{Error}_{3}(h)\right| \leq c\left(1+S_{0}^{p}\right) \sup _{i}\left(\delta_{i} \bar{\sigma} \sqrt{T-t_{i}}\right)^{3}$. 
Proof. The proof is similar to that of Theorem 2.1, except that the equality 2.2 is replaced by a second order Taylor expansion. It gives

$$
\begin{aligned}
\mathbb{E}\left[e^{-\int_{0}^{T} r_{s} d s} h\left(S_{T}^{(y, \delta)}-K\right)\right]=\mathbb{E}\left[e^{-\int_{0}^{T} r_{s} d s} h\left(\bar{S}_{T}^{(y, \delta)}-K\right)\right] \\
-\sum_{i=1}^{n} \hat{\delta}_{i} \mathbb{E}\left[e^{-\int_{0}^{T} r_{s} d s} h^{\prime}\left(\bar{S}_{T}^{(y, \delta)}-K\right)\left(\frac{M_{T}}{M_{t_{i}}}-1\right)\right] \\
+\frac{1}{2} \mathbb{E}\left[e^{-\int_{0}^{T} r_{s} d s} h^{\prime \prime}\left(\bar{S}_{T}^{(y, \delta)}-K\right)\left(\sum_{i=1}^{n} \hat{\delta}_{i}\left[\frac{M_{T}}{M_{t_{i}}}-1\right]\right)^{2}\right] \\
+\operatorname{Error}_{3}(h)
\end{aligned}
$$

where $\operatorname{Error}_{3}(h) \leq_{c}\left(1+S_{0}^{p}\right) \sup _{i}\left(\delta_{i}\left\|\frac{M_{T}}{M_{t_{i}}}-1\right\|_{4}\right)^{3}$. Then, the announced estimate on $\operatorname{Error}_{3}(h)$ easily follows by using (2.3).

Comparing with the expansion in Theorem 2.1, it remains to transform the new contribution with the factor $\frac{1}{2}$. This term is equal to

$$
\begin{aligned}
& \mathbb{E}\left[e^{-\int_{0}^{T} r_{s} d s} h^{\prime \prime}\left(\bar{S}_{T}^{(y, \delta)}-K\right)\left(\sum_{i=1}^{n} \hat{\delta}_{i} \frac{M_{T}}{M_{t_{i}}}-\sum_{i=1}^{n} \hat{\delta}_{i}\right)^{2}\right] \\
= & \sum_{1 \leq i, j \leq n} \hat{\delta}_{i} \hat{\delta}_{j} \mathbb{E}\left[e^{-\int_{0}^{T} r_{s} d s} h^{\prime \prime}\left(\bar{S}_{T}^{(y, \delta)}-K\right) \frac{M_{T}}{M_{t_{i}}} \frac{M_{T}}{M_{t_{j}}}\right] \\
& -2\left(\sum_{j=1}^{n} \hat{\delta}_{j}\right) \sum_{i=1}^{n} \hat{\delta}_{i} \mathbb{E}\left[e^{-\int_{0}^{T} r_{s} d s} h^{\prime \prime}\left(\bar{S}_{T}^{(y, \delta)}-K\right) \frac{M_{T}}{M_{t_{i}}}\right] \\
& +\left(\sum_{j=1}^{n} \hat{\delta}_{j}\right)^{2} \mathbb{E}\left[e^{-\int_{0}^{T} r_{s} d s} h^{\prime \prime}\left(\bar{S}_{T}^{(y, \delta)}-K\right)\right] \\
:= & \mathcal{T}_{1}+\mathcal{T}_{2}+\mathcal{T}_{3} .
\end{aligned}
$$

We handle separately each of the three terms above.

- Term $\mathcal{T}_{1}$. We proceed analogously to the equality (2.7) by transforming this term via different changes of probability measure. Indeed, note that $\frac{M_{T}}{M_{t_{i}}} \frac{M_{T}}{M_{t_{j}}}=\exp \left(\int_{0}^{T} \sigma_{s}\left(\mathbf{1}_{t_{i} \leq s \leq T}+\mathbf{1}_{t_{j} \leq s \leq T}\right) d W_{s}-\frac{1}{2} \int_{t_{i}}^{T}\left[\sigma_{s}\left(\mathbf{1}_{t_{i} \leq s \leq T}+\right.\right.\right.$ $\left.\left.\left.\mathbf{1}_{t_{j} \leq s \leq T}\right)\right]^{2} d s\right) \exp \left(\int_{t_{i} \vee t_{j}}^{T} \sigma_{s}^{2} d s\right.$ ) defines (up to the second exponential factor) a change of measure $\mathbb{Q}^{i, j}$ under which $\left(W_{t}-\int_{0}^{t} \sigma_{s}\left(\mathbf{1}_{t_{i} \leq s \leq T}+\mathbf{1}_{t_{j} \leq s \leq T}\right) d s\right)_{t \geq 0}$ is a Brownian motion. It means that $\bar{S}_{T}^{(y, \delta)}$ under $\mathbb{Q}^{i, j}$ has the same law 
as $\pi_{0, n} S_{T} e^{\int_{t_{i}}^{T} \sigma_{s}^{2} d s+\int_{t_{j}}^{T} \sigma_{s}^{2} d s}-\sum_{l=1}^{n} \hat{\delta}_{l}$ under $\mathbb{Q}$. Thus, we obtain

$$
\begin{aligned}
\mathcal{T}_{1} & =\sum_{1 \leq i, j \leq n} \hat{\delta}_{i} \hat{\delta}_{j} \mathbb{E}\left[e^{-\int_{0}^{T} r_{s} d s} h^{\prime \prime}\left(\pi_{0, n} e^{\int_{t_{i}}^{T} \sigma_{s}^{2} d s+\int_{t_{j}}^{T} \sigma_{s}^{2} d s} S_{T}-\sum_{l=1}^{n} \hat{\delta}_{l}-K\right)\right] e^{\int_{t_{i} \vee t_{j}}^{T} \sigma_{s}^{2} d s} \\
& =\left.\sum_{1 \leq i, j \leq n} \hat{\delta}_{i} \hat{\delta}_{j} \partial_{k}^{2} \mathbb{E}\left[e^{-\int_{0}^{T} r_{s} d s} h\left(\pi_{0, n} e^{\int_{t_{i}}^{T} \sigma_{s}^{2} d s+\int_{t_{j}}^{T} \sigma_{s}^{2} d s} S_{T}-k\right)\right]\right|_{k=K^{(y, \delta)}} e^{\int_{t_{i} \vee t_{j}}^{T} \sigma_{s}^{2} d s},
\end{aligned}
$$

using (2.6) at the last line.

- Term $\mathcal{T}_{2}$. Similarly, we obtain

$$
\mathcal{T}_{2}=-\left.2\left(\sum_{j=1}^{n} \hat{\delta}_{j}\right) \sum_{i=1}^{n} \hat{\delta}_{i} \partial_{k}^{2} \mathbb{E}\left[e^{-\int_{0}^{T} r_{s} d s} h\left(\pi_{0, n} e^{\int_{t_{i}}^{T} \sigma_{s}^{2} d s} S_{T}-k\right)\right]\right|_{k=K^{(y, \delta)}}
$$

- Term $\mathcal{T}_{3}$. Clearly, we have

$$
\mathcal{T}_{3}=\left.\left(\sum_{j=1}^{n} \hat{\delta}_{j}\right)^{2} \partial_{k}^{2} \mathbb{E}\left[e^{-\int_{0}^{T} r_{s} d s} h\left(\pi_{0, n} S_{T}-k\right)\right]\right|_{k=K^{(y, \delta)}} .
$$

The theorem is proved.

\subsection{Expansion results for call payoff}

We now extend the previous results from smooth functions $h$ to the call option function $h(x)=x_{+}$, using a regularization argument that is quite standard. However one has to be carefull with the error estimates since they depend on $h^{\prime \prime}$ or $h^{\prime \prime \prime}$ in the previous case of smooth functions. To safely pass to the limit, we impose the non-degenaracy condition $(\mathbf{E})$ on the model. The assumption (D) enables us to get error estimates uniform in $t_{1}$, as $t_{1}$ goes to 0 .

We first precise the derivatives of $\mathrm{Call}^{B S}(x, k)$ with respect to the strike $k$. We have

$$
\begin{gathered}
\partial_{k} \operatorname{Call}^{B S}(x, k)=-e^{-\int_{0}^{T} r_{s} d s} \mathcal{N}\left(d_{-}(x, k)\right), \\
\partial_{k}^{2} \operatorname{Call}^{B S}(x, k)=\frac{e^{-\int_{0}^{T} r_{s} d s}}{k \sqrt{2 \pi \int_{0}^{T} \sigma_{s}^{2} d s}} e^{-\frac{1}{2} d_{-}^{2}(x, k)}
\end{gathered}
$$


and

$$
\partial_{k}^{3} \operatorname{Call}^{B S}(x, k)=\frac{e^{-\int_{0}^{T} r_{s} d s}}{k^{2} \sqrt{2 \pi \int_{0}^{T} \sigma_{s}^{2} d s}} e^{-\frac{1}{2} d_{-}^{2}(x, k)}\left(\frac{d_{-}(x, k)}{\sqrt{\int_{0}^{T} \sigma_{s}^{2} d s}}-1\right) .
$$

We now state our main results, giving a first and second order formula for the price of a Call option written on a multidividend asset (a third order formula is given in Subsection 2.4).

Theorem 2.3. Assume (D) and (E). We have

$$
\begin{aligned}
& \mathbb{E}\left(e^{-\int_{0}^{T} r_{s} d s}\left(S_{T}^{(y, \delta)}-K\right)_{+}\right) \\
= & \operatorname{Call}^{B S}\left(\pi_{0, n} S_{0}, K^{(y, \delta)}\right) \\
& +\sum_{i=1}^{n} \hat{\delta}_{i}\left(\partial_{k} \operatorname{Call}^{B S}\left(\pi_{0, n} S_{0} e^{\int_{t_{i}}^{T} \sigma_{s}^{2} d s}, K^{(y, \delta)}\right)-\partial_{k} \operatorname{Call}^{B S}\left(\pi_{0, n} S_{0}, K^{(y, \delta)}\right)\right) \\
& + \text { Error }_{2}(\text { Call }),
\end{aligned}
$$

with $\mid \operatorname{Error}_{2}($ Call $) \mid \leq c \sup _{i}\left(\frac{\delta_{i}}{S_{0}} \sqrt{1-\frac{t_{i}}{T}}\right)^{2} S_{0} \bar{\sigma} \sqrt{T}$.

The result below states a second order approximation result.

Theorem 2.4. Assume (D) and (E). We have

$$
\begin{aligned}
& \mathbb{E}\left(e^{-\int_{0}^{T} r_{s} d s}\left(S_{T}^{(y, \delta)}-K\right)_{+}\right) \\
= & \operatorname{Call}^{B S}\left(\pi_{0, n} S_{0}, K^{(y, \delta)}\right) \\
& +\sum_{i=1}^{n} \hat{\delta}_{i}\left(\partial_{k} \operatorname{Call}^{B S}\left(\pi_{0, n} S_{0} e^{\int_{t_{i}}^{T} \sigma_{s}^{2} d s}, K^{(y, \delta)}\right)-\partial_{k} \operatorname{Call}^{B S}\left(\pi_{0, n} S_{0}, K^{(y, \delta)}\right)\right) \\
& +\frac{1}{2}\left(\sum_{1 \leq i, j \leq n} \hat{\delta}_{i} \hat{\delta}_{j} e^{\int_{t_{i} \vee t_{j}}^{T} \sigma_{s}^{2} d s} \partial_{k}^{2} \mathrm{Call}^{B S}\left(\pi_{0, n} S_{0} e^{\int_{t_{i}}^{T} \sigma_{s}^{2} d s+\int_{t_{j}}^{T} \sigma_{s}^{2} d s}, K^{(y, \delta)}\right)\right. \\
& -2\left(\sum_{j=1}^{n} \hat{\delta}_{j}\right) \sum_{i=1}^{n} \hat{\delta}_{i} \partial_{k}^{2} \mathrm{Call}^{B S}\left(\pi_{0, n} S_{0} e^{\int_{t_{i}}^{T} \sigma_{s}^{2} d s}, K^{(y, \delta)}\right) \\
& \left.+\left(\sum_{j=1}^{n} \hat{\delta}_{j}\right)^{2} \partial_{k}^{2} \mathrm{Call}^{B S}\left(\pi_{0, n} S_{0}, K^{(y, \delta)}\right)\right)+\operatorname{Error}_{3}(\mathrm{Call})
\end{aligned}
$$

with $\mid \operatorname{Error}_{3}($ Call $) \mid \leq c \sup _{i}\left(\frac{\delta_{i}}{S_{0}} \sqrt{1-\frac{t_{i}}{T}}\right)^{3} S_{0} \bar{\sigma} \sqrt{T}$. 
To state the error estimates, we have taken a specific form which allows to assert that our approximation error is of order one or two w.r.t. $\sup _{i} \delta_{i} / S_{0}$. This is especially clear for At-The-Money options, for which $\pi_{0, n} S_{0} e^{-\int_{0}^{T} q_{s} d s}=$ $K^{(y, \delta)} e^{-\int_{0}^{T} r_{s} d s}$. In that case, using the Brenner-Subrahmanyam approximation [BS88] Call $\left.{ }^{B S}(x, k)\right|_{k=x}=\frac{1}{\sqrt{2 \pi}} x(\sqrt{v}+o(v))$ as $v=\int_{0}^{T} \sigma_{s}^{2} d s$ goes to 0 , we obtain that the relative ATM error is bounded by $c \sup _{i}\left(\frac{\delta_{i}}{S_{0}} \sqrt{1-\frac{t_{i}}{T}}\right)^{2}$ (in Theorem 2.3p or $c \sup _{i}\left(\frac{\delta_{i}}{S_{0}} \sqrt{1-\frac{t_{i}}{T}}\right)^{3}$ (in Theorem 2.4p. This indicates that the relative accuracy of our approximation depends mainly of the ratio $\sup _{i} \delta_{i} / S_{0}$ and not much of the other parameters. This is confirmed by the numerical results (see Section 4).

The results for put option are simply obtained by replacing the Call ${ }^{B S}($. function by the $\operatorname{Put}^{B S}$ (.) function. Then we observe that these approximations verify the Call-Put parity relation.

\subsection{Proof of Theorems 2.3 and 2.4}

The sketch of the proof is the following: we take a sequence of smooth functions $\left(h_{N}\right)_{N}$ converging to $h(x)=x_{+}$in a suitable sense. Then, the proof is divided in two steps.

1. First, we prove that the expansion terms computed with $h_{N}$ converge to those computed with $h$.

2. Second, we estimate the limsup of the error terms Error $_{2}\left(h_{N}\right)$ and Error $_{3}\left(h_{N}\right)$ as $N$ goes to infinity.

In this subsection, we only give details regarding Step 1. Step 2, involving Malliavin calculus, is much more technical. We postpone it to the next section.

The justification of the Step 1 relies on the following Lemma.

Lemma 2.1. Assume $(\mathbf{E})$, take $\alpha>0$ and $k>0$. Consider a sequence of measurable functions $\left(h_{N}\right)_{N \geq 1}$ and $h$ having a polynomial growth uniformly in $N$, i.e. for some constants $C>0$ and $p>0$ we have $\sup _{x \in \mathbb{R}} \frac{\left|h_{N}(x)\right|+|h(x)|}{\left(1+|x|^{p}\right)} \leq C$.

i) Then, the functions $k \mapsto \mathbb{E}\left[h_{N}\left(\alpha S_{T}-k\right)\right]$ and $k \mapsto \mathbb{E}\left[h\left(\alpha S_{T}-k\right)\right]$ are infinitely continuously differentiable on $] 0, \infty[$. 
ii) In addition, assume that $h_{N}$ converges almost everywhere to $h$ as $N$ goes to infinity. Then, for any $m \in \mathbb{N}$, we have

$$
\lim _{N \rightarrow \infty} \partial_{k}^{m} \mathbb{E}\left[h_{N}\left(\alpha S_{T}-k\right)\right]=\partial_{k}^{m} \mathbb{E}\left[h\left(\alpha S_{T}-k\right)\right]
$$

Proof. Under (E), the law of $S_{T}$ has an explicit density w.r.t. the Lebesgue measure. It gives

$$
\begin{aligned}
\mathbb{E}\left[h\left(\alpha S_{T}-k\right)\right] & =\int_{\mathbb{R}} h\left(\alpha \frac{S_{0}}{D_{T}} e^{x \sqrt{\int_{0}^{T} \sigma_{s}^{2} d s}-\frac{1}{2} \int_{0}^{T} \sigma_{s}^{2} d s}-k\right) \frac{\exp \left(-x^{2} / 2\right)}{\sqrt{2 \pi}} d x \\
& =\int_{-k}^{\infty} h(z) p(z+k) d z
\end{aligned}
$$

where $p(u)=\mathbf{1}_{u>0} \frac{\exp \left(-\left[\log \left(u D_{T} /\left(\alpha S_{0}\right)\right)+\frac{1}{2} \int_{0}^{T} \sigma_{s}^{2} d s\right]^{2} /\left[2 \int_{0}^{T} \sigma_{s}^{2} d s\right]\right)}{u \sqrt{2 \pi \int_{0}^{T} \sigma_{s}^{2} d s}}$. It is easy to check that $p$ is infinitely continuously differentiable on $] 0, \infty[$ and that its derivatives at $u=0$ are equal to 0 . In addition, for any $m \in \mathbb{N}$, we have $\int_{0}^{\infty}\left|\partial_{u}^{m} p(u)\right|(1+$ $\left.|u|^{p}\right) d u<\infty$. These properties easily imply that $\mathbb{E}\left[h\left(\alpha S_{T}-k\right)\right]$ is smooth w.r.t. $k$ (i.e. statement $i$ )) and that

$$
\partial_{k}^{m} \mathbb{E}\left[h\left(\alpha S_{T}-k\right)\right]=\int_{-k}^{\infty} h(z) \partial_{z}^{m} p(z+k) d z .
$$

From this representation and by an application of the dominated convergence theorem, statement ii) readily follows.

A sequence of functions $\left(h_{N}\right)_{N \geq 1}$ satisfying $\left(\mathbf{H}_{3}\right)$ and converging to $h(x)=x_{+}$. For $N \in \mathbb{N}^{*}$, take $h_{N}$ defined by $h_{N}(x)=\int_{-\infty}^{x} \int_{-\infty}^{u} N(1-$ $N|v|)_{+} d v d u$ : each function $h_{N}$ satisfies $\left(\mathbf{H}_{3}\right)$ and it is easy to check the following properties

i) $h_{N}(x)=h_{N}^{\prime}(x)=0$ for $x \leq-1 / N$,

ii) $h_{N}(x)=x$ and $h_{N}^{\prime}(x)=1$ for $x \geq 1 / N$,

iii) $0 \leq h_{N}^{\prime}(x) \leq 1$,

iv) $\left(h_{N}\right)_{N}$ converges uniformly to $h$ as $N \rightarrow \infty$. 
Owing to the above uniform convergence, we have

$$
\begin{aligned}
\lim _{N \rightarrow \infty} \mathbb{E}\left(e^{-\int_{0}^{T} r_{s} d s} h_{N}\left(S_{T}^{(y, \delta)}-K\right)\right) & =\mathbb{E}\left(e^{-\int_{0}^{T} r_{s} d s}\left(S_{T}^{(y, \delta)}-K\right)_{+}\right), \\
\lim _{N \rightarrow \infty} \mathbb{E}\left(e^{-\int_{0}^{T} r_{s} d s} h_{N}\left(\pi_{0, n} S_{T}-K^{(y, \delta)}\right)\right) & =\mathbb{E}\left(e^{-\int_{0}^{T} r_{s} d s}\left(\pi_{0, n} S_{T}-K^{(y, \delta)}\right)_{+}\right) \\
& =\operatorname{Call}^{B S}\left(\pi_{0, n} S_{0}, K^{(y, \delta)}\right)
\end{aligned}
$$

using the Black-Scholes formula (1.8) for the last equality. Moreover, using Lemma 2.1, we obtain for any $\alpha>0$

$$
\begin{aligned}
\lim _{N \rightarrow \infty} \partial_{k} \mathbb{E}\left(e^{-\int_{0}^{T} r_{s} d s} h_{N}\left(\alpha S_{T}-k\right)\right) & =\partial_{k} \mathbb{E}\left(e^{-\int_{0}^{T} r_{s} d s}\left(\alpha S_{T}-k\right)_{+}\right) \\
& =\partial_{k} \operatorname{Call}^{B S}\left(\alpha S_{0}, k\right) .
\end{aligned}
$$

Thus, we can apply Theorem 2.1 with $h_{N}$ and pass to the limit as $N$ goes to infinity. It gives the expansion of Theorem 2.3, with

$$
\lim _{N \rightarrow \infty} \operatorname{Error}_{2}\left(h_{N}\right)=\text { Error }_{2}(\text { Call }) .
$$

However, the upper bounds on Error $_{2}\left(h_{N}\right)$ given in Theorem 2.1 involve $h_{N}^{\prime \prime}$ and it does not enable us to pass to the limit on the error estimates. In the next section, we prove specific estimates using Malliavin calculus:

Proposition 2.1. Assume (D) and (E). Then, we have

$$
\left|\operatorname{Error}_{2}\left(h_{N}\right)\right| \leq c \sup _{i}\left(\frac{\delta_{i}}{S_{0}} \sqrt{1-\frac{t_{i}}{T}}\right)^{2} S_{0} \bar{\sigma} \sqrt{T},
$$

uniformly in $N$. Consequently, the same estimate applies to Error $_{2}(\mathrm{Call})$.

Using the above result, the proof of Theorem 2.3 is complete.

Similarly, for the second derivative, we have

$$
\lim _{N \rightarrow \infty} \partial_{k}^{2} \mathbb{E}\left(e^{-\int_{0}^{T} r_{s} d s} h_{N}\left(\alpha S_{T}-k\right)\right)=\partial_{k}^{2} \operatorname{Call}^{B S}\left(\alpha S_{0}, k\right)
$$

Analogously to Proposition 2.1, we have

Proposition 2.2. Assume (D) and (E). Then, we have

$$
\left|\operatorname{Error}_{3}\left(h_{N}\right)\right| \leq c \sup _{i}\left(\frac{\delta_{i}}{S_{0}} \sqrt{1-\frac{t_{i}}{T}}\right)^{3} S_{0} \bar{\sigma} \sqrt{T},
$$

uniformly in $N$. Consequently, the same estimate applies to Error $_{3}(\mathrm{Call})$.

Thus, we complete the proof of Theorem 2.4 as for Theorem 2.3. 


\subsection{Extension to the third-order approximation price formula}

Using similar techniques we can state a third order formula. We leave the details of the proof to the reader.

Theorem 2.5. Assume (D) and (E). We have

$$
\begin{aligned}
& \mathbb{E}\left(e^{-\int_{0}^{T} r_{s} d s}\left(S_{T}^{(y, \delta)}-K\right)_{+}\right) \\
& =\text {Call }^{B S}\left(\pi_{0, n} S_{0}, K^{(y, \delta)}\right) \\
& +\sum_{i=1}^{n} \hat{\delta}_{i}\left(\partial_{k} \operatorname{Call}^{B S}\left(\pi_{0, n} S_{0} e^{\int_{t_{i}}^{T} \sigma_{s}^{2} d s}, K^{(y, \delta)}\right)-\partial_{k} \operatorname{Call}^{B S}\left(\pi_{0, n} S_{0}, K^{(y, \delta)}\right)\right) \\
& +\frac{1}{2}\left(\sum_{1 \leq i, j \leq n} \hat{\delta}_{i} \hat{\delta}_{j} e^{\int_{t_{i} \vee t_{j}}^{T} \sigma_{s}^{2} d s} \partial_{k}^{2} \operatorname{Call}^{B S}\left(\pi_{0, n} S_{0} e^{\int_{t_{i}}^{T} \sigma_{s}^{2} d s+\int_{t_{j}}^{T} \sigma_{s}^{2} d s}, K^{(y, \delta)}\right)\right. \\
& -2\left(\sum_{j=1}^{n} \hat{\delta}_{j}\right) \sum_{i=1}^{n} \hat{\delta}_{i} \partial_{k}^{2} \operatorname{Call}^{B S}\left(\pi_{0, n} S_{0} e^{\int_{t_{i}}^{T} \sigma_{s}^{2} d s}, K^{(y, \delta)}\right) \\
& \left.+\left(\sum_{j=1}^{n} \hat{\delta}_{j}\right)^{2} \partial_{k}^{2} \operatorname{Call}^{B S}\left(\pi_{0, n} S_{0}, K^{(y, \delta)}\right)\right) \\
& +\frac{1}{6}\left(\sum_{1 \leq i, j, l \leq n} \hat{\delta}_{i} \hat{\delta}_{j} \hat{\delta}_{l} e^{\int_{t_{i} \vee t_{j}}^{T} \sigma_{s}^{2} d s+\int_{t_{i} \vee t_{l}}^{T} \sigma_{s}^{2} d s+\int_{t_{j} \vee t_{l}}^{T} \sigma_{s}^{2} d s} \times\right. \\
& \partial_{k}^{3} \operatorname{Call}^{B S}\left(\pi_{0, n} S_{0} e^{\int_{t_{i}}^{T} \sigma_{s}^{2} d s+\int_{t_{j}}^{T} \sigma_{s}^{2} d s+\int_{t_{l}}^{T} \sigma_{s}^{2} d s}, K^{(y, \delta)}\right) \\
& -3\left(\sum_{j=1}^{n} \hat{\delta}_{j}\right) \sum_{1 \leq i, j \leq n} \hat{\delta}_{i} \hat{\delta}_{j} e^{\int_{t_{i} \vee t_{j}}^{T} \sigma_{s}^{2} d s} \partial_{k}^{3} \operatorname{Call}^{B S}\left(\pi_{0, n} S_{0} e^{\int_{t_{i}}^{T} \sigma_{s}^{2} d s+\int_{t_{j}}^{T} \sigma_{s}^{2} d s}, K^{(y, \delta)}\right) \\
& +3\left(\sum_{j=1}^{n} \hat{\delta}_{j}\right)^{2} \sum_{i=1}^{n} \hat{\delta}_{i} \partial_{k}^{3} \operatorname{Call}^{B S}\left(\pi_{0, n} S_{0} e^{\int_{t_{i}}^{T} \sigma_{s}^{2} d s}, K^{(y, \delta)}\right) \\
& \left.-\left(\sum_{j=1}^{n} \hat{\delta}_{j}\right)^{3} \partial_{k}^{3} \operatorname{Call}^{B S}\left(\pi_{0, n} S_{0}, K^{(y, \delta)}\right)\right) \\
& +\operatorname{Error}_{4}(\text { Call })
\end{aligned}
$$




\subsection{Extension to the approximation of the Delta}

Adapting our methodology we can also derive several expansion formulas for the delta of a Call option on a multidividend asset. We choose to present only the second order approximation formula.

Let us first fix some extra notations. With the convention that $t_{0}=0$ we set

$$
\begin{gathered}
\pi_{i, n}^{\Delta}:=\pi_{i, n} e^{\int_{t_{i}}^{T} \sigma_{s}^{2} d s}, \quad \forall 0 \leq i \leq n, \\
\hat{\delta}_{i}^{\Delta}:=\delta_{i} \pi_{i, n}^{\Delta} \frac{D_{t_{i}}}{D_{T}}, \quad \forall 1 \leq i \leq n \quad \text { and } \quad K^{(y, \delta, \Delta)}:=K+\sum_{i=1}^{n} \hat{\delta}_{i}^{\Delta} .
\end{gathered}
$$

Theorem 2.6. Assume (D) and (E). Let $\Delta=\partial_{S_{0}} \mathbb{E}\left(e^{-\int_{0}^{T} r_{s} d s}\left(S_{T}^{(y, \delta)}-K\right)_{+}\right)$ be the Delta of the Call option of strike $K$ on the multidividend asset. We have

$$
\begin{aligned}
\Delta=\pi_{0, n}\{ & \partial_{x} \operatorname{Call}^{B S}\left(\pi_{0, n} S_{0}, K^{(y, \delta, \Delta)}\right) \\
& +\sum_{i=1}^{n} \hat{\delta}_{i}^{\Delta}\left(\partial_{k, x}^{2} \operatorname{Call}^{B S}\left(\pi_{0, n} S_{0} e^{\int_{t_{i}}^{T} \sigma_{s}^{2} d s}, K^{(y, \delta, \Delta)}\right)\right. \\
& \left.-\partial_{k, x}^{2} \operatorname{Call}^{B S}\left(\pi_{0, n} S_{0}, K^{(y, \delta, \Delta)}\right)\right) \\
+ & \frac{1}{2}\left(\sum_{1 \leq i, j \leq n} \hat{\delta}_{i}^{\Delta} \hat{\delta}_{j}^{\Delta} e^{\int_{t_{i} \vee t_{j}}^{T} \sigma_{s}^{2} d s} \partial_{k, k, x}^{3} \operatorname{Call}^{B S}\left(\pi_{0, n} S_{0} e^{\int_{t_{i}}^{T} \sigma_{s}^{2} d s+\int_{t_{j}}^{T} \sigma_{s}^{2} d s}, K^{(y, \delta, \Delta)}\right)\right. \\
& -2\left(\sum_{j=1}^{n} \hat{\delta}_{j}^{\Delta}\right) \sum_{i=1}^{n} \hat{\delta}_{i}^{\Delta} \partial_{k, k, x}^{3} \operatorname{Call}^{B S}\left(\pi_{0, n} S_{0} e^{\int_{t_{i}}^{T} \sigma_{s}^{2} d s}, K^{(y, \delta, \Delta)}\right) \\
& \left.\left.+\left(\sum_{j=1}^{n} \hat{\delta}_{j}^{\Delta}\right)^{2} \partial_{k, k, x}^{3} \operatorname{Call}^{B S}\left(\pi_{0, n} S_{0}, K^{(y, \delta, \Delta)}\right)\right)\right\} \\
+ & \text { Error }_{3}(\text { Digital }),
\end{aligned}
$$

with $\mid \operatorname{Error}_{3}($ Digital $) \mid \leq c \sup _{i}\left(\frac{\delta_{i}}{S_{0}} \sqrt{1-\frac{t_{i}}{T}}\right)^{3}$.

Remark 2.1. The third order error is denoted Error $_{3}$ (Digital) because, up to multiplicative constants, the Delta is of the form, $\Delta=\mathbb{E}\left(e^{-\int_{0}^{T} r_{s} d s} \mathbf{1}_{S_{T}^{(y, \delta, \Delta)}>K}\right)$, that is the price of a Digital Call option with $S^{(y, \delta, \Delta)}$ as an asset to be described in the following sketch of proof.

Remark 2.2. We recall that $\partial_{x} \operatorname{Call}^{B S}(x, k)=e^{-\int_{0}^{T} q_{s} d s} \mathcal{N}\left(d_{+}(x, k)\right)$. The other greeeks are easily computed from (2.10), 2.11). We skip details. 
Proof. We only give the main lines. Details can be treated adapting the proofs of Theorems 2.2 and 2.4 .

Step 1. Taking into account Lemma 1.1. the pathwise derivative of $S_{T}^{(y, \delta)}$ w.r.t. $S_{0}$ is $\pi_{0, n} \frac{S_{T}}{S_{0}}=\frac{\pi_{0, n}}{D_{T}} M_{T}$. Thus, interchanging derivation and expectation,

$$
\Delta=\frac{\pi_{0, n}}{D_{T}} \mathbb{E}\left(e^{-\int_{0}^{T} r_{s} d s} \mathbf{1}_{S_{T}^{(y, \delta)}>K} M_{T}\right) .
$$

Again we interpret $M_{T}$ as a change of measure on $\mathcal{F}_{T}$. Under the new induced measure $\mathbb{Q}^{0}, \bar{W}_{t}=W_{t}-\int_{0}^{t} \sigma_{s} d s$ is a brownian motion. Then $S_{T}^{(y, \delta)}$ under $\mathbb{Q}^{0}$ has the same law as

$$
S_{T}^{(y, \delta, \Delta)}:=\pi_{0, n} S_{T} e^{\int_{0}^{T} \sigma_{s}^{2} d s}-\sum_{i=1}^{n} \delta_{i} \pi_{i, n} \frac{S_{T}}{S_{t_{i}}} e^{\int_{t_{i}}^{T} \sigma_{s}^{2} d s}=\pi_{0, n}^{\Delta} S_{T}-\sum_{i=1}^{n} \delta_{i} \pi_{i, n}^{\Delta} \frac{S_{T}}{S_{t_{i}}},
$$

under $\mathbb{Q}$. Thus,

$$
\Delta=\frac{\pi_{0, n}}{D_{T}} \mathbb{E}\left(e^{-\int_{0}^{T} r_{s} d s} h\left(S_{T}^{(y, \delta, \Delta)}-K\right)\right),
$$

with $h(x)=\mathbf{1}_{x>0}$.

Step 2. From 2.16), we see that the evaluation of the Delta is reduced to that of the price of a digital Call written on an asset $S^{(y, \delta, \Delta)}$ with new dividend parameters $\left(\pi_{i, n}^{\Delta}\right)_{i}$ (compare Lemma 1.1 and 2.15)). Then, the derivation of an approximation formula is similar to what we have done in Theorem 2.2 and 2.4. Briefly, we take a sequence of smooth functions $\left(h_{N}:=\frac{1}{2}(\tanh (N .)+1)\right)_{N}$ converging to $h$ almost everywhere, and we apply 
Theorem 2.2 and Lemma 2.1. It gives

$$
\begin{aligned}
\Delta= & \frac{\pi_{0, n}}{D_{T}}\left[\mathbb{E}\left[e^{-\int_{0}^{T} r_{s} d s} h\left(\pi_{0, n}^{\Delta} S_{T}-K^{(y, \delta, \Delta)}\right)\right]\right. \\
+ & \sum_{i=1}^{n} \hat{\delta}_{i}^{\Delta}\left(\left.\partial_{k} \mathbb{E}\left[e^{-\int_{0}^{T} r_{s} d s} h\left(\pi_{0, n}^{\Delta} e^{\int_{t_{i}}^{T} \sigma_{s}^{2}} S_{T}-k\right)\right]\right|_{k=K}(y, \delta, \Delta)\right. \\
& \left.\quad-\left.\partial_{k} \mathbb{E}\left[e^{-\int_{0}^{T} r_{s} d s} h\left(\pi_{0, n}^{\Delta} S_{T}-k\right)\right]\right|_{k=K^{(y, \delta, \Delta)}}\right) \\
+ & \frac{1}{2}\left(\left.\sum_{1 \leq i, j \leq n} \hat{\delta}_{i}^{\Delta} \hat{\delta}_{j}^{\Delta} \partial_{k}^{2} \mathbb{E}\left[e^{-\int_{0}^{T} r_{s} d s} h\left(\pi_{0, n}^{\Delta} e^{\int_{t_{i}}^{T} \sigma_{s}^{2} d s+\int_{t_{j}}^{T} \sigma_{s}^{2} d s} S_{T}-k\right)\right]\right|_{k=K^{(y, \delta, \Delta)}} e^{\int_{t_{i} \vee t_{j}}^{T} \sigma_{s}^{2} d s}\right. \\
& -\left.2\left(\sum_{j=1}^{n} \hat{\delta}_{j}^{\Delta}\right) \sum_{i=1}^{n} \hat{\delta}_{i}^{\Delta} \partial_{k}^{2} \mathbb{E}\left[e^{-\int_{0}^{T} r_{s} d s} h\left(\pi_{0, n}^{\Delta} e^{\int_{t_{i}}^{T} \sigma_{s}^{2} d s} S_{T}-k\right)\right]\right|_{k=K^{(y, \delta, \Delta)}} \\
& \left.\left.+\left.\left(\sum_{j=1}^{n} \hat{\delta}_{j}^{\Delta}\right)^{2} \partial_{k}^{2} \mathbb{E}\left[e^{-\int_{0}^{T} r_{s} d s} h\left(\pi_{0, n}^{\Delta} S_{T}-k\right)\right]\right|_{k=K^{(y, \delta, \Delta)}}\right)\right]+\lim _{N \rightarrow \infty} \operatorname{Error}_{3}\left(h_{N}\right) .
\end{aligned}
$$

Similarly to Proposition 2.2, it is possible to prove that

$$
\left|\operatorname{Error}_{3}\left(h_{N}\right)\right| \leq c \sup _{i}\left(\frac{\delta_{i}}{S_{0}} \sqrt{1-\frac{t_{i}}{T}}\right)^{3},
$$

uniformly in $N$, using $\left|h_{N}\right|_{\infty}=1$ (see Remark 3.1). This gives the upper bound for Error $_{3}($ Digital).

Step 3. It remains to relate the correction terms to the Black-Scholes formula. Actually, for any multiplicative constant $\alpha>0$ and any positive strike $k$, we have

$$
\begin{aligned}
\frac{\pi_{0, n}}{D_{T}} \mathbb{E}\left[e^{-\int_{0}^{T} r_{s} d s} \mathbf{1}_{\pi_{0, n}^{\Delta} \alpha S_{T}>k}\right] & =\pi_{0, n} e^{-\int_{0}^{T} q_{s} d s} \mathcal{N}\left(d_{-}\left(\pi_{0, n}^{\Delta} \alpha S_{0}, k\right)\right) \\
& =\pi_{0, n} e^{-\int_{0}^{T} q_{s} d s} \mathcal{N}\left(d_{+}\left(\pi_{0, n} \alpha S_{0}, k\right)\right) \\
& =\pi_{0, n} \partial_{x} \operatorname{Call}^{B S}\left(\alpha \pi_{0, n} S_{0}, k\right)
\end{aligned}
$$

Then, by successive differentiation w.r.t. $k$, we obtain the announced formula. 


\section{Proof of Propositions 2.1 and 2.2}

In the proof of Theorems 2.1 and 2.2 , we have obtained that

$$
\begin{aligned}
& \operatorname{Error}_{2}\left(h_{N}\right)=\mathbb{E}\left(e^{-\int_{0}^{T} r_{s} d s}\left(\sum_{i=1}^{n} \hat{\delta}_{i}\left(\frac{M_{T}}{M_{t_{i}}}-1\right)\right)^{2} \int_{0}^{1}(1-\lambda) h_{N}^{\prime \prime}\left(F_{T}^{\lambda}-K\right) d \lambda\right), \\
& \operatorname{Error}_{3}\left(h_{N}\right)=\mathbb{E}\left(e^{-\int_{0}^{T} r_{s} d s}\left(\sum_{i=1}^{n} \hat{\delta}_{i}\left(\frac{M_{T}}{M_{t_{i}}}-1\right)\right)^{3} \int_{0}^{1} \frac{(1-\lambda)^{2}}{2} h_{N}^{\prime \prime \prime}\left(F_{T}^{\lambda}-K\right) d \lambda\right)
\end{aligned}
$$

where $\forall 0 \leq \lambda \leq 1$ we define

$$
F_{T}^{\lambda}:=\bar{S}_{T}^{(y, \delta)}-\lambda \sum_{i=1}^{n} \delta_{i} \pi_{i, n} \frac{D_{t_{i}}}{D_{T}}\left(\frac{M_{T}}{M_{t_{i}}}-1\right) .
$$

\subsection{Technical results from Malliavin calculus}

Our aim is to provide tight error estimates on $\sup _{N}\left|\operatorname{Error}_{2}\left(h_{N}\right)\right|$ and $\sup _{N}\left|\operatorname{Error}_{3}\left(h_{N}\right)\right|$, using $\left|h_{N}^{\prime}\right|_{\infty}=1$. For this, we use the Malliavin calculus integration by parts to transform the above expectations. It requires the use of several lemmas stated hereafter, that will be proved in the next subsection. The results deal with the Malliavin estimates of the random variable $F_{T}^{\lambda}$ and $\left(\frac{M_{T}}{M_{t_{i}}}-1\right)$. Regarding to Malliavin calculus related to the onedimensional Brownian motion $W$, we freely adopt the notation from [Nua06]. For instance, the first Malliavin derivative of a random variable $F$ is the $H=L^{2}([0, T], d t)$-valued process denoted by $\mathcal{D} F=\left(\mathcal{D}_{t} F\right)_{0 \leq t \leq T}$. The second derivative takes values in $H^{\otimes_{2}}$ and is denoted by $\mathcal{D}^{2} F=\left(\mathcal{D}_{s, t}^{2} F\right)_{0 \leq s, t \leq T}$, and so on. If the scalar product on $H^{\otimes_{k}}$ is denoted by $\langle., .\rangle_{H^{\otimes k}}$, then the Malliavin covariance matrix of $F$ is defined by $\gamma_{F}=\langle\mathcal{D} F, \mathcal{D} F\rangle_{H}$. We freely use the notation $\mathbb{D}^{k, p}(k \geq 1, p \geq 1)$ for the space of $k$-times Malliavin differentiable random variables (with derivatives in $L_{p}$ ) and related $\|\cdot\|_{k, p}$-norms (see [Nua06, Section 1.2]). We set $\mathbb{D}^{k, \infty}=\cap_{p \geq 1} \mathbb{D}^{k, p}$ and $\mathbb{D}^{\infty}=\cap_{k \geq 1} \mathbb{D}^{k, \infty}$.

Lemma 3.1. For all $p \geq 1$, all $0 \leq \lambda \leq 1, F_{T}^{\lambda}$ is in $\mathbb{D}^{\infty}$ and

$$
\begin{array}{ll}
\sup _{t \leq T}\left\|\mathcal{D}_{t}\left(F_{T}^{\lambda}\right)\right\|_{p} \leq c_{p} S_{0} \bar{\sigma}, & \sup _{t, r \leq T}\left\|\mathcal{D}_{t, r}^{2}\left(F_{T}^{\lambda}\right)\right\|_{p} \leq_{c_{p}} S_{0} \bar{\sigma}^{2}, \\
\sup _{t, r, s \leq T}\left\|\mathcal{D}_{t, r, s}^{3}\left(F_{T}^{\lambda}\right)\right\|_{p} \leq_{c_{p}} S_{0} \bar{\sigma}^{3} . &
\end{array}
$$


Lemma 3.2. Assume (D) and (E). We have for all $0 \leq \lambda \leq 1, \gamma_{F_{T}^{\lambda}}^{-1} \in$ $\cap_{p \geq 1} L^{p}(\Omega)$. In addition,

$$
\forall p \geq 1, \quad \forall 0 \leq \lambda \leq 1, \quad\left\|\gamma_{F_{T}^{\lambda}}^{-1}\right\|_{2, p} \leq_{c_{p}} \frac{1}{S_{0}^{2} \underline{\sigma}^{2} T}
$$

Lemma 3.3. Let $\left(N_{t}\right)_{0 \leq t \leq T}$ be a Brownian martingale with a bounded bracket, and assume that $J_{T}=N_{T}-\frac{1}{2}\langle N\rangle_{T}$ is in $\mathbb{D}^{2, \infty}$. Then for all $r \geq 1$, one has

$$
\left\|e^{J_{T}}-1\right\|_{2, r} \leq_{c_{r}}\left\|J_{T}\right\|_{2,2 r}\left(1+\left\|J_{T}\right\|_{2,4 r}+\left\|J_{T}\right\|_{1,8 r}^{2}\right) e^{2 r \sup _{\omega}\langle N\rangle_{T}} .
$$

We are now in a position to complete the proof of Propositions 2.1 and 2.2. Let us start with Error $_{2}\left(h_{N}\right)$ : by Fubini's theorem, it is equal to

$$
\sum_{1 \leq i, j \leq n} e^{-\int_{0}^{T} r_{s} d s} \hat{\delta}_{i} \hat{\delta}_{j} \int_{0}^{1}(1-\lambda) \mathbb{E}\left[\left(\frac{M_{T}}{M_{t_{i}}}-1\right)\left(\frac{M_{T}}{M_{t_{j}}}-1\right) h_{N}^{\prime \prime}\left(F_{T}^{\lambda}-K\right)\right] d \lambda .
$$

We now control (uniformly in $\lambda$ and $N$ ) the above expectations. To remove the singularity problem of $h_{N}^{\prime \prime}$, we apply an integration by parts of Malliavin calculus. Indeed, from [Nua06, Proposition 2.1.4], one knows that for $1 \leq$ $i, j \leq n$ and $0 \leq \lambda \leq 1$ there exists $H_{i j}^{1, \lambda} \in \mathbb{D}^{\infty}$, depending only on $F_{T}^{\lambda}$ and $\left(\frac{M_{T}}{M_{t_{i}}}-1\right)\left(\frac{M_{T}}{M_{t_{j}}}-1\right)$, such that

$$
\mathbb{E}\left[\left(\frac{M_{T}}{M_{t_{i}}}-1\right)\left(\frac{M_{T}}{M_{t_{j}}}-1\right) h_{N}^{\prime \prime}\left(F_{T}^{\lambda}-K\right)\right]=\mathbb{E}\left[h_{N}^{\prime}\left(F_{T}^{\lambda}-K\right) H_{i j}^{1, \lambda}\right] .
$$

This is justified by the fact that $\left(\frac{M_{T}}{M_{t_{i}}}-1\right)\left(\frac{M_{T}}{M_{t_{j}}}-1\right) \in \mathbb{D}^{\infty}, F_{T}^{\lambda}$ is in $\mathbb{D}^{\infty}$ and is non degenerate (Lemma 3.2) under the assumption (E). Our task then becomes to find an upper bound, uniformly in $\lambda, i, j$, for $\left\|H_{i j}^{1, \lambda}\right\|_{p}$, for all $p$. Using the discussion in [Nua06, p.102] we have

$$
\left\|H_{i j}^{1, \lambda}\right\|_{p} \leq_{c_{p}}\left\|\gamma_{F_{T}^{\lambda}}^{-1}\right\|_{1,4 p}\left\|\mathcal{D} .\left(F_{T}^{\lambda}\right)\right\|_{1,4 p}\left\|\left(\frac{M_{T}}{M_{t_{i}}}-1\right)\left(\frac{M_{T}}{M_{t_{j}}}-1\right)\right\|_{1,2 p} .
$$

Considering Lemma 3.2 it remains to estimate the two last terms of the r.h.s. above. By definition, we have

$$
\left\|\mathcal{D} .\left(F_{T}^{\lambda}\right)\right\|_{1, q}^{q}=\mathbb{E}\left|\left(\int_{0}^{T}\left|\mathcal{D}_{r}\left(F_{T}^{\lambda}\right)\right|^{2} d r\right)^{1 / 2}\right|^{q}+\mathbb{E}\left(\int_{0}^{T} \int_{0}^{T}\left|\mathcal{D}_{s, r}^{2}\left(F_{T}^{\lambda}\right)\right|^{2} d r d s\right)^{q / 2} .
$$

Then by standard inequalities combined with Lemma 3.1. we get for any $q$

$$
\left\|\mathcal{D} .\left(F_{T}^{\lambda}\right)\right\|_{1, q} \leq_{c_{q}} S_{0} \bar{\sigma} \sqrt{T}
$$


On the other hand, using Hölder type inequalities on $\|\cdot\|_{k, p}$-norms (see [Nua06, Proposition 1.5.6]), we have

$$
\left\|\left(\frac{M_{T}}{M_{t_{i}}}-1\right)\left(\frac{M_{T}}{M_{t_{j}}}-1\right)\right\|_{1, q} \leq_{c_{1, q}}\left\|\frac{M_{T}}{M_{t_{i}}}-1\right\|_{1,2 q}\left\|\frac{M_{T}}{M_{t_{j}}}-1\right\|_{1,2 q} .
$$

In order to apply Lemma 3.3, we define the Brownian martingale $N_{t}=$ $\int_{0}^{t} \sigma_{s} \mathbf{1}_{t_{i} \leq s \leq T} d W_{s}$ which bracket is bounded by $\bar{\sigma}^{2}\left(T-t_{i}\right)$. Using the notation of Lemma 3.3, notice that $e^{J_{T}}=e^{N_{T}-\frac{1}{2}\langle N\rangle_{T}}=\frac{M_{T}}{M_{t_{i}}}$. Clearly $\left\|J_{T}\right\|_{2, r} \leq_{c_{r}}$ $\bar{\sigma} \sqrt{T-t_{i}}$. Then from Lemma 3.3 , it readily follows that

$$
\left\|\frac{M_{T}}{M_{t_{i}}}-1\right\|_{2, r} \leq_{c_{r}} \bar{\sigma} \sqrt{T-t_{i}}
$$

In particular, it gives $\left\|\left(\frac{M_{T}}{M_{t_{i}}}-1\right)\left(\frac{M_{T}}{M_{t_{j}}}-1\right)\right\|_{1, q} \leq_{c_{q}} \bar{\sigma}^{2} \sqrt{T-t_{i}} \sqrt{T-t_{j}}$.

We combine the latter inequality with (3.6), (3.7), Lemma 3.2 and we obtain for any $i, j, p$

$$
\left\|H_{i j}^{1, \lambda}\right\|_{p} \leq_{c_{p}} \bar{\sigma} \frac{\sqrt{T-t_{i}} \sqrt{T-t_{j}}}{S_{0} \sqrt{T}}
$$

uniformly in $\lambda$. Plugging this estimate into (3.5) (and using $\left|h_{N}^{\prime}\right|_{\infty}=1$ ) leads to

$$
\left|\mathbb{E}\left[\left(\frac{M_{T}}{M_{t_{i}}}-1\right)\left(\frac{M_{T}}{M_{t_{j}}}-1\right) h_{N}^{\prime \prime}\left(F_{T}^{\lambda}-K\right)\right]\right| \leq_{c} \bar{\sigma} \frac{\sqrt{T-t_{i}} \sqrt{T-t_{j}}}{S_{0} \sqrt{T}} .
$$

In view of (3.4), we have proved that $\left|\operatorname{Error}_{2}\left(h_{N}\right)\right| \leq_{c} \frac{\sup _{i}\left(\delta_{i} \sqrt{T-t_{i}}\right)^{2}}{S_{0} \sqrt{T}} \bar{\sigma}$. Proposition 2.1 is proved.

The proof of Proposition 2.2 is very similar and we only give the main intermediate estimates. Analogously to the identity (3.5), we have

$$
\begin{aligned}
\operatorname{Error}_{3}(h, N)= & \sum_{1 \leq i, j, l \leq n} e^{-\int_{0}^{T} r_{s} d s} \hat{\delta}_{i} \hat{\delta}_{j} \hat{\delta}_{l} \int_{0}^{1} \frac{(1-\lambda)^{2}}{2} \\
& \times \mathbb{E}\left[\left(\frac{M_{T}}{M_{t_{i}}}-1\right)\left(\frac{M_{T}}{M_{t_{j}}}-1\right)\left(\frac{M_{T}}{M_{t_{l}}}-1\right) h_{N}^{\prime \prime \prime}\left(F_{T}^{\lambda}-K\right)\right] d \lambda \\
= & \sum_{1 \leq i, j, l \leq n} e^{-\int_{0}^{T} r_{s} d s} \hat{\delta}_{i} \hat{\delta}_{j} \hat{\delta}_{l} \int_{0}^{1} \frac{(1-\lambda)^{2}}{2} \mathbb{E}\left[h_{N}^{\prime}\left(F_{T}^{\lambda}-K\right) H_{i j l}^{2, \lambda}\right] \lambda .
\end{aligned}
$$


Furthermore, applying the general estimates from [Nua06, p.102] combined with (3.8), we obtain

$$
\begin{aligned}
& \left|\operatorname{Error}_{3}(h, N)\right| \leq_{c} \sum_{1 \leq i, j, l \leq n} \hat{\delta}_{i} \hat{\delta}_{j} \hat{\delta}_{l} \sup _{\lambda \in[0,1]}\left\|H_{i j k}^{2, \lambda}\right\|_{1} \\
& \leq_{c} \sum_{1 \leq i, j, l \leq n} \hat{\delta}_{i} \hat{\delta}_{j} \hat{\delta}_{l} \sup _{\lambda \in[0,1]}\left(\left\|\gamma_{F_{T}^{\lambda}}^{-1}\right\|_{2,8}^{2}\left\|\mathcal{D} .\left(F_{T}^{\lambda}\right)\right\|_{2,8}^{2}\left\|\left(\frac{M_{T}}{M_{t_{i}}}-1\right)\left(\frac{M_{T}}{M_{t_{j}}}-1\right)\left(\frac{M_{T}}{M_{t_{l}}}-1\right)\right\|_{2,2}\right) \\
& \leq_{c} \sum_{1 \leq i, j, l \leq n} \hat{\delta}_{i} \hat{\delta}_{j} \hat{\delta}_{l} \frac{1}{\left(S_{0}^{2} \underline{\sigma}^{2} T\right)^{2}}\left(S_{0} \bar{\sigma} \sqrt{T}\right)^{2} \bar{\sigma}^{3} \sqrt{T-t_{i}} \sqrt{T-t_{j}} \sqrt{T-t_{l}} \\
& \leq_{c} \sup _{i}\left(\hat{\delta}_{i} \sqrt{T-t_{i}}\right)^{3} \frac{\bar{\sigma}}{S_{0}^{2} T} .
\end{aligned}
$$

We are finished.

Remark 3.1. In the proof of Theorem 2.6 we have to control uniformly in $N$, $\mid$ Error $_{3}\left(h_{N}\right) \mid$, with $\left(h_{N}\right)_{N}$ approximating $h(x)=\mathbf{1}_{x>0}$, and satisfying $\left|h_{N}\right|_{\infty}=1$. Compared to the proof of Proposition 2.2, we have to use

$$
\mathbb{E}\left[\left(\frac{M_{T}}{M_{t_{i}}}-1\right)\left(\frac{M_{T}}{M_{t_{j}}}-1\right)\left(\frac{M_{T}}{M_{t_{l}}}-1\right) h_{N}^{\prime \prime \prime}\left(F_{T}^{\lambda}-K\right)\right]=\mathbb{E}\left[h_{N}\left(F_{T}^{\lambda}-K\right) H_{i j l}^{3, \lambda}\right] .
$$

Indeed we cannot have a uniform control on $h_{N}^{\prime}$ here. Similarly to the proofs above, we have

$$
\begin{aligned}
\sup _{\lambda \in[0,1]}\left\|H_{i j k}^{3, \lambda}\right\|_{1} & \leq_{c}\left\|\gamma_{F_{T}^{\lambda}}^{-1}\right\|_{3,16}^{3}\left\|\mathcal{D} .\left(F_{T}^{\lambda}\right)\right\|_{3,16}^{3}\left\|\left(\frac{M_{T}}{M_{t_{i}}}-1\right)\left(\frac{M_{T}}{M_{t_{j}}}-1\right)\left(\frac{M_{T}}{M_{t_{l}}}-1\right)\right\|_{3,2} \\
& \leq_{c} \frac{1}{\left(S_{0}^{2} \underline{\sigma}^{2} T\right)^{3}}\left(S_{0} \bar{\sigma} \sqrt{T}\right)^{3} \bar{\sigma}^{3} \sqrt{T-t_{i}} \sqrt{T-t_{j}} \sqrt{T-t_{l}}
\end{aligned}
$$

and thus the error estimate (2.17) stated in Theorem 2.6. 


\subsection{Proof of technical lemmas}

Proof of Lemma 3.1. Take a fixed $\lambda$. As $F_{T}^{\lambda}$ is an affine function of the lognormal variables $\frac{M_{T}}{M_{t_{i}}}$ 's (see $(3.3)$ ) it is clear that $F_{T}^{\lambda} \in \mathbb{D}^{\infty}$. We have

$$
\begin{aligned}
\mathcal{D}_{t}\left(F_{T}^{\lambda}\right) & =S_{0} \pi_{0, n} \frac{M_{T}}{D_{T}} \sigma_{t} \mathbf{1}_{t \leq T}-\lambda \sum_{i=1}^{n} \delta_{i} \pi_{i, n} \frac{D_{t_{i}}}{D_{T}} \frac{M_{T}}{M_{t_{i}}} \sigma_{t} \mathbf{1}_{t_{i}<t \leq T}, \\
\mathcal{D}_{t, r}^{2}\left(F_{T}^{\lambda}\right) & =S_{0} \pi_{0, n} \frac{M_{T}}{D_{T}} \sigma_{t} \sigma_{r} \mathbf{1}_{t, r \leq T}-\lambda \sum_{i=1}^{n} \delta_{i} \pi_{i, n} \frac{D_{t_{i}}}{D_{T}} \frac{M_{T}}{M_{t_{i}}} \sigma_{t} \sigma_{r} \mathbf{1}_{t_{i}<t, r \leq T}, \\
\mathcal{D}_{t, r, s}^{3}\left(F_{T}^{\lambda}\right) & =S_{0} \pi_{0, n} \frac{M_{T}}{D_{T}} \sigma_{t} \sigma_{r} \sigma_{s} \mathbf{1}_{t, r, s \leq T}-\lambda \sum_{i=1}^{n} \delta_{i} \pi_{i, n} \frac{D_{t_{i}}}{D_{T}} \frac{M_{T}}{M_{t_{i}}} \sigma_{t} \sigma_{r} \sigma_{s} \mathbf{1}_{t_{i}<t, r, s \leq T} .
\end{aligned}
$$

Standard computations (using $\sup _{i} \delta_{i} \leq c_{\delta} S_{0}$ ) lead easily to the announced estimates.

Proof of Lemma 3.2. It is enough to consider the case $p \geq 2$. Take $\lambda \in[0,1]$. Step 1. We first estimate $\gamma_{F_{T}^{\lambda}}^{-1}$ in $L^{p}$. We have

$$
\begin{aligned}
\gamma_{F_{T}^{\lambda}} & =\left\|\mathcal{D} F_{T}^{\lambda}\right\|_{H}^{2}=\int_{0}^{T}\left|\mathcal{D}_{s}\left(F_{T}^{\lambda}\right)\right|^{2} d s \\
& \geq S_{T}^{2} \underline{\sigma}^{2} \int_{0}^{T}\left|\pi_{0, n}-\lambda \sum_{i=1}^{n} \pi_{i, n} \frac{\delta_{i}}{S_{t_{i}}} \mathbf{1}_{t_{i} \leq s \leq T}\right|^{2} d s \\
& \geq S_{T}^{2} \underline{\sigma}^{2} \pi_{0, n}^{2}\left(t_{1}+\left|1-\lambda \frac{\delta_{1}}{\left(1-y_{1}\right) S_{t_{1}}}\right|^{2}\left(T \mathbf{1}_{n=1}+t_{2} \mathbf{1}_{n>1}-t_{1}\right)\right)
\end{aligned}
$$

Thus, it is clear that

$$
\gamma_{F_{T}^{\lambda}} \geq S_{T}^{2} \underline{\sigma}^{2} \pi_{0, n}^{2} t_{1}
$$

inducing that $\gamma_{F_{T}^{\lambda}}$ is invertible and its inverse is in any $L^{p}$. Now, our aim is to estimate the $L^{p}$-norm of $\gamma_{F_{T}^{\lambda}}^{-1}$ uniformly in $t_{1} \leq 1$. For this, we define the event

$$
\mathcal{A}_{1}=\left\{\frac{1}{1+\beta} \geq \frac{\delta_{1}}{\left(1-y_{1}\right) S_{t_{1}}}\right\}
$$

where the parameter $\beta$ will be set at a positive value close to 0 . Then, on this event, we have

$$
1-\lambda \frac{\delta_{1}}{\left(1-y_{1}\right) S_{t_{1}}} \geq 1-\frac{\lambda}{1+\beta} \geq \frac{\beta}{1+\beta}>0 .
$$


Thus, on $\mathcal{A}_{1}$, we obtain

$$
\begin{aligned}
\frac{\gamma_{F_{T}^{\lambda}}}{S_{T}^{2} \underline{\underline{\sigma}}^{2} \pi_{0, n}^{2}} & \geq t_{1}+\frac{\beta^{2}}{(1+\beta)^{2}}\left(T \mathbf{1}_{n=1}+t_{2} \mathbf{1}_{n>1}-t_{1}\right) \\
& \geq \frac{\beta^{2}}{(1+\beta)^{2}}\left(T \mathbf{1}_{n=1}+t_{2} \mathbf{1}_{n>1}\right) \geq \frac{\beta^{2}}{(1+\beta)^{2}}(1 \wedge T)
\end{aligned}
$$

using if $n>1$ that $t_{2} \geq 1$.

We now estimate $\mathbb{P}\left(\mathcal{A}_{1}^{c}\right)$ by leveraging the assumption $S_{0}\left(1-y_{1}\right)<\delta_{1}$. Using that $S_{t_{1}}$ has a lognormal distribution, we obtain

$$
\begin{aligned}
\mathbb{P}\left(\mathcal{A}_{1}^{c}\right) & =\mathbb{P}\left(M_{t_{1}}<D_{t_{1}} \frac{\delta_{1}(1+\beta)}{S_{0}\left(1-y_{1}\right)}\right) \\
& =\mathcal{N}\left(\frac{1}{\left(\int_{0}^{t_{1}} \sigma_{s}^{2} d s\right)^{1 / 2}}\left[\log \left(D_{t_{1}}\right)+\frac{1}{2} \int_{0}^{t_{1}} \sigma_{s}^{2} d s+\log \left(\frac{\delta_{1}(1+\beta)}{S_{0}\left(1-y_{1}\right)}\right)\right]\right) .
\end{aligned}
$$

Choose $\beta$ close to 0 enough to ensure that $\log \left(\frac{\delta_{1}(1+\beta)}{S_{0}\left(1-y_{1}\right)}\right)=C_{\beta}<0$. Then, using $\mathcal{N}(x) \leq \exp \left(-x_{-}^{2} / 2\right)$ for any $x$, we deduce

$$
\mathbb{P}\left(\mathcal{A}_{1}^{c}\right) \leq \exp \left(-\frac{1}{2 \bar{\sigma}^{2} t_{1}}\left[\left(|r-q|_{\infty} t_{1}+\frac{1}{2} \bar{\sigma}^{2} t_{1}+C_{\beta}\right)_{-}\right]^{2}\right) \leq_{c_{p}} 1 \wedge\left(\bar{\sigma}^{2} t_{1}\right)^{p}
$$

for any $p>0$.

Finally, bringing together our different estimates, we deduce

$$
0 \leq \gamma_{F_{T}^{\lambda}}^{-1} \leq \frac{S_{T}^{-2}}{\pi_{0, n}^{2} \underline{\sigma}^{2}(1 \wedge T)} \frac{(1+\beta)^{2}}{\beta^{2}} \mathbf{1}_{\mathcal{A}_{1}}+\frac{S_{T}^{-2}}{\pi_{0, n}^{2} \underline{\sigma}^{2} t_{1}} \mathbf{1}_{\mathcal{A}_{1}^{c}}
$$

By Hölder inequalities, together with the fact that $S_{T}^{-1} \in \cap_{p \geq 1} L^{p}$, we obtain

$$
\left\|\gamma_{F_{T}^{\lambda}}^{-1}\right\|_{p} \leq_{c} \frac{1}{S_{0}^{2}}\left(\frac{1}{\underline{\sigma}^{2}(1 \wedge T)}+\frac{\bar{\sigma}^{2}}{{\underline{\sigma^{2}}}^{2}}\right) \leq_{c} \frac{1}{S_{0}^{2} \underline{\sigma}^{2} T},
$$

possibly changing the value of the generic constant $c$ at the last inequality. This proves the first statement of the Lemma.

Step 2. We turn to estimate the Malliavin derivatives of $\gamma_{F_{T}^{\lambda}}^{-1}$. By the chain rule, we obtain

$$
\mathcal{D}_{s}\left(\gamma_{F_{T}^{\lambda}}^{-1}\right)=-\frac{\mathcal{D}_{s} \gamma_{F_{T}^{\lambda}}}{\gamma_{F_{T}^{\lambda}}^{2}} \quad \text { and } \quad \mathcal{D}_{s, t}^{2}\left(\gamma_{F_{T}^{\lambda}}^{-1}\right)=-\frac{\mathcal{D}_{s, t}^{2} \gamma_{F_{T}^{\lambda}}}{\gamma_{F_{T}^{\lambda}}^{2}}+2 \frac{\mathcal{D}_{s} \gamma_{F_{T}^{\lambda}} \mathcal{D}_{t} \gamma_{F_{T}^{\lambda}}}{\gamma_{F_{T}^{\lambda}}^{3}}
$$


On the one hand, by definition of the $\|\cdot\|_{2,2 r}$-norms, we have

$$
\begin{aligned}
& \left\|\gamma_{F_{T}^{\lambda}}^{-1}\right\|_{2, p}^{p}=\mathbb{E}\left(\gamma_{F_{T}^{\lambda}}^{-p}\right)+\mathbb{E}\left\|\mathcal{D}\left(\gamma_{F_{T}^{\lambda}}^{-1}\right)\right\|_{H}^{p}+\mathbb{E}\left\|\mathcal{D}^{2}\left(\gamma_{F_{T}^{\lambda}}^{-1}\right)\right\|_{H^{\otimes_{2}}}^{p} \\
& \quad \leq_{c} \mathbb{E}\left(\gamma_{F_{T}^{\lambda}}^{-p}\right)+\mathbb{E}\left(\gamma_{F_{T}^{\lambda}}^{-2 p}\left\|\mathcal{D} \gamma_{F_{T}^{\lambda}}\right\|_{H}^{p}\right)+\mathbb{E}\left(\gamma_{F_{T}^{\lambda}}^{-2 p}\left\|\mathcal{D}^{2} \gamma_{F_{T}^{\lambda}}\right\|_{H^{\otimes_{2}}}^{p}\right)+\mathbb{E}\left(\gamma_{F_{T}^{\lambda}}^{-3 p}\left\|\mathcal{D} \gamma_{F_{T}^{\lambda}}\right\|_{H}^{2 p}\right),
\end{aligned}
$$

that is

$$
\begin{aligned}
\left\|\gamma_{F_{T}^{\lambda}}^{-1}\right\|_{2, p} \leq_{c} \| & \gamma_{F_{T}^{\lambda}}^{-1}\left\|_{p}+\right\| \gamma_{F_{T}^{\lambda}}^{-1}\left\|_{4 p}^{2}\right\|\left\|\mathcal{D} \gamma_{F_{T}^{\lambda}}\right\|_{H} \|_{2 p} \\
& +\left\|\gamma_{F_{T}^{\lambda}}^{-1}\right\|_{4 p}^{2}\|\| \mathcal{D}^{2} \gamma_{F_{T}^{\lambda}}\left\|_{H^{\otimes_{2}}}\right\|_{2 p}+\left\|\gamma_{F_{T}^{\lambda}}^{-1}\right\|_{6 p}^{3}\|\| \mathcal{D} \gamma_{F_{T}^{\lambda}}\left\|_{H}\right\|_{4 p}^{2}
\end{aligned}
$$

One the other hand, using Minkowski and Hölder inequalities combined with Lemma 3.3, from 3.10$)$ we derive

$$
\begin{aligned}
\|\| \mathcal{D} \gamma_{F_{T}^{\lambda}}\left\|_{H}\right\|_{2 p} & =\left\|\int_{0}^{T}\left|\mathcal{D}_{t} \gamma_{F_{T}^{\lambda}}\right|^{2} d t\right\|_{p}^{1 / 2} \leq\left(\int_{0}^{T}\left\|\mathcal{D}_{t} \gamma_{F_{T}^{\lambda}}\right\|_{2 p}^{2} d t\right)^{1 / 2} \\
& \leq\left(\int_{0}^{T}\left\|\int_{0}^{T} 2 \mathcal{D}_{s} F_{T}^{\lambda} \mathcal{D}_{t, s}^{2} F_{T}^{\lambda} d s\right\|_{2 p}^{2} d t\right)^{1 / 2} \\
& \leq\left(\int_{0}^{T}\left(\int_{0}^{T} 2\left\|\mathcal{D}_{s} F_{T}^{\lambda}\right\|_{4 p}\left\|\mathcal{D}_{t, s}^{2} F_{T}^{\lambda}\right\|_{4 p} d s\right)^{2} d t\right)^{1 / 2} \leq_{c} S_{0}^{2} \bar{\sigma}^{3} T^{3 / 2} .
\end{aligned}
$$

Similarly, we obtain

$$
\|\| \mathcal{D}^{2} \gamma_{F_{T}^{\lambda}}\left\|_{H^{\otimes_{2}}}\right\|_{2 p} \leq\left(\int_{0}^{T} \int_{0}^{T}\left(\int_{0}^{T}\left\|\mathcal{D}_{t, r}^{2}\left[\left(\mathcal{D}_{s} F_{T}^{\lambda}\right)^{2}\right]\right\|_{2 p} d s\right)^{2} d t d r\right)^{1 / 2} \leq_{c} S_{0}^{2} \bar{\sigma}^{4} T^{2} .
$$

Plugging the above inequalities and the estimate 3.12 into 3.13 yields

$$
\begin{aligned}
\left\|\gamma_{F_{T}^{\lambda}}^{-1}\right\|_{2, p} \leq & \leq_{c}\left\|\gamma_{F_{T}^{\lambda}}^{-1}\right\|_{p}+\left\|\gamma_{F_{T}^{\lambda}}^{-1}\right\|_{4 p}^{2}\|\| \mathcal{D} \gamma_{F_{T}^{\lambda}}\left\|_{H}\right\|_{2 p} \\
& +\left\|\gamma_{F_{T}^{\lambda}}^{-1}\right\|_{4 p}^{2}\|\| \mathcal{D}^{2} \gamma_{F_{T}^{\lambda}}\left\|_{H^{\otimes_{2}}}\right\|_{2 p}+\left\|\gamma_{F_{T}^{\lambda}}^{-1}\right\|_{6 p}^{3}\|\| \mathcal{D} \gamma_{F_{T}^{\lambda}}\left\|_{H}\right\|_{4 p}^{2} . \\
\leq & \frac{1}{S_{0}^{2} \frac{\sigma^{2} T}{1}}\left(1+\frac{1}{S_{0}^{2} \underline{\sigma}^{2} T}\left(S_{0}^{2} \bar{\sigma}^{3} T^{3 / 2}+S_{0}^{2} \bar{\sigma}^{4} T^{2}\right)+\frac{1}{\left(S_{0}^{2} \underline{\sigma}^{2} T\right)^{2}}\left(S_{0}^{2} \bar{\sigma}^{4} T^{2}\right)^{2}\right) \\
\leq & \leq \frac{1}{S_{0}^{2} \underline{\sigma}^{2} T} .
\end{aligned}
$$

The proof is complete.

Proof of Lemma 3.3. The Taylor formula yields

$$
e^{J_{T}}-1=J_{T} \int_{0}^{1} e^{u J_{T}} d u
$$


Using Hölder and Minkowski inequalities, we obtain

$$
\left\|e^{J_{T}}-1\right\|_{2, r} \leq_{c}\left\|J_{T}\right\|_{2,2 r} \int_{0}^{1}\left\|e^{u J_{T}}\right\|_{2,2 r} d u
$$

For any $0 \leq u \leq 1$, we have

$$
\mathcal{D}_{s} e^{u J_{T}}=u e^{u J_{T}} \mathcal{D}_{s} J_{T} \quad \text { and } \quad \mathcal{D}_{s, t}^{2} e^{u J_{T}}=e^{u J_{T}}\left(u \mathcal{D}_{s, t}^{2} J_{T}+u^{2} D_{s} J_{T} D_{t} J_{T}\right) .
$$

Then, by definition of the $\|\cdot\|_{2,2 r}$-norms, we obtain

$$
\begin{aligned}
\left\|e^{u J_{T}}\right\|_{2,2 r}^{2 r} & =\mathbb{E}\left(\left[e^{u J_{T}}\right]^{2 r}\right)+\mathbb{E}\left\|\mathcal{D} e^{u J_{T}}\right\|_{H}^{2 r}+\mathbb{E}\left\|\mathcal{D}^{2} e^{u J_{T}}\right\|_{H^{\otimes_{2}}}^{2 r} \\
& \leq \mathbb{E}\left(e^{2 r u J_{T}}\right)+\mathbb{E}\left(e^{2 r u J_{T}}\left\|\mathcal{D} J_{T}\right\|_{H}^{2 r}\right)+\mathbb{E}\left(e^{2 r u J_{T}}\left(\left\|\mathcal{D}^{2} J_{T}\right\|_{H^{\otimes_{2}}}+\left\|\mathcal{D} J_{T}\right\|_{H}^{2}\right)^{2 r}\right) \\
& \leq{ }_{c}\left\|e^{u J_{T}}\right\|_{4 r}^{2 r}\left(1+\left\|J_{T}\right\|_{2,4 r}^{2 r}+\left\|J_{T}\right\|_{1,8 r}^{4 r}\right) .
\end{aligned}
$$

Finally, since $\left(e^{p u N_{t}-\frac{1}{2}\langle p u N\rangle_{t}}\right)_{t}$ defines an exponential martingale (for any fixed $p)$, one has

$\left\|e^{u J_{T}}\right\|_{p}^{p}=\mathbb{E}\left[e^{p u\left(N_{T}-\frac{1}{2}\langle N\rangle_{T}\right)}\right]=\mathbb{E}\left[e^{p u N_{T}-\frac{1}{2}\langle p u N\rangle_{T}+\frac{1}{2}\langle N\rangle_{T}\left(-p u+(p u)^{2}\right)}\right] \leq e^{\frac{p^{2}}{2} \sup _{\omega}\langle N\rangle_{T}}$.

Plugging this estimate into (3.15) and 3.14), we get the announced result.

\section{Numerical experiments}

In all our tests we use as benchmark a Monte Carlo price computed with $2.10^{9}$ drawings, and control variates (column "Monte Carlo" in the tables). The control variates consist in European options with the same parameters except that $\delta \equiv 0$ (see the discussion after Lemma 1.1). In the tables the numbers between parentheses in the Monte Carlo columns refer to the half width of the $95 \%$ confidence intervall around the computed prices.

We wish first to compare our results with the ones of recent papers in the literature (namely [BGS03, VN06, VW09]). In Table 1, the abbrevations EG3, VNRE, VN1000, VW and BGS refer respectively to our method with the order three formula, the method of Vellekoop and Nieuwenhuis with Richardson Extrapolation, their method without extrapolation and 1000 time steps (both in [VN06]), the method in [VW09] and the method in [BGS03]. The example is the one treated in these last three papers: till time maturity $T=7.0$ we have 7 dividend payment dates $0<t_{1}<\ldots<t_{7}<T$ with 
$t_{i+1}-t_{i}=1$ for all $1 \leq i<7$. We test the cases $t_{1}=0.1,0.5$ and 0.9 . The successive $\delta_{i}$ 's are $6,6.5,7,7.5,8,8$ and 8 . We have $y \equiv 0$. The coefficients $\left(r_{t}\right)_{t},\left(q_{t}\right)_{t}$ and $\left(\sigma_{t}\right)_{t}$ are constant, with $r=6 \%, q=0 \%$ and $\sigma=25 \%$. We take $S_{0}=100$ and test the strikes $K=70,100$ and 130 .

insert Table 1 about here

These tests show that the accuracy of our method is better than the one of VN1000 and BGS and similar to the one of VW. However the VNRE method seems to be the most accurate.

But note that, for $n$ dividend payment dates, the number of terms to compute in our order two and three formulae are respectively

$$
\frac{(n+2)(n+3)}{2} \text { and } \quad \frac{(n+2)\left(n^{2}+7 n+12\right)}{6} .
$$

Thus, the number of terms computed for EG3 in Table1 is 165, which requires a small computational time. Concerning the VNRE method the maximal number of time steps is 64000, which is fairly demanding. See also the discussion p13 in [VW09]: 2187 evaluations of the Black-Scholes formula and any of its derivatives are computed to achieve the prices reported in Table 1 . In other words, from the computational point of view, our approach is very competitive, compared to other existing methods.

We now test the sensitivity to the parameters of the precision of our option pricers. As indicated by Theorems 2.3, 2.4 and 2.5, the error should increase with volatility, time maturity and the amplitude of the $\delta_{i}$ 's.

In Table 2 we have $r=6 \%, q=0 \%, S_{0}=100, n=3$, and $y_{i}=0.02$ and $\delta_{i}=2$ for all $1 \leq i \leq n$. We have $t_{1}=0.5, t_{i+1}-t_{i}=1$ for all $1 \leq i<n$ and $T=3.0$. We successively test $\sigma=15 \%, 25 \%, 45 \%$, and compute the prices with the formulae at order one, two and three (respectively EG1, EG2 and EG3) for various strikes. Under each price we report the corresponding implied volatility (expressed in \%).

\section{Insert Table 2 about here}

As the volatility $\sigma$ increases we observe a loss of accuracy on the prices computed with EG1, while for EG2 and EG3 the accuracy remains nearly the same. This suggests that our method is quite robust to variations of the volatility. 
In Table 3 we set $\sigma=25 \%$, the other parameters as in Table 2, and test the influence of the amplitude of the $\delta_{i}$ 's. We take $\delta_{i}=\delta$ for all $1 \leq i \leq n$ and test the values $\delta=2,6,10$.

Insert Table 3 about here

With $\delta \equiv 2$ the results of EG1, EG2 and EG3 are accurate up to one basis point on implied volatilities (even if EG1 seems to be a bit less accurate on the prices themselves). With $\delta \equiv 6$ both EG2 and EG3 match the implied volatities, but we observe a slightly difference of accuracy on the prices. With $\delta \equiv 10$ only EG3 still performs well to match prices and implied volatilities. Note that, as expected the solvers are always more accurate at the money.

Finally, in Table 4 we investigate the influence of $n$, keeping $\sigma=25 \%$ constant, and the other parameters as in Table 2, except $\delta \equiv 4$. We choose $n=3,5,10$, which is related to testing the influence of the maturity $T=n$.

\section{Insert Table 4 about here}

With $n=3$ the solvers EG2 and EG3 perfectly match the implied volatilities. The solver EG1 is accurate up to $2 \mathrm{bp}$ on implied volatilities, which is generally sufficient for calibration purposes. As expected, with $n=10$ a loss of accuracy can be observed (both on prices and implied volatilities). Even with EG3 the implied volatilities can fail to match the ones corresponding to Monte Carlo prices. Some computed prices are slightly outside the Monte Carlo confidence interval (especially for in the money options).

Note that similar tests show no significative influence of the parameters $y_{i}$ on the results: for $\sigma=0.25$ and the other parameters as in Table 2, EG2 and EG3 both match the implied volatility with 0 bp error, whatever the value of the $y_{i}$ 's.

Note also that we have used our Monte Carlo simulations to estimate the probabilities that $S_{T}^{(y, \delta)}<0$. Indeed, with the affine type dividend model there is no guarantee that this never occurs. The numerical results show that this probability increases with $\delta$ and $n$ (see Tables 5 and 6). For $n=10$ this probability is larger than $2 \%$ (in the results of Table 1 this estimated probability is also about $2 \%$ : indeed the dividends are of high amplitude and $n=7)$. This suggests that the dividend model itself has to be refined as $S^{(y, \delta)}$ is close to zero. 
Insert Tables 5 and 6 about here

\section{Conclusion}

In this work, we have derived approximation formulae for the vanilla option prices written on an asset paying discrete dividends, under lognormality assumptions. Numerical tests show that the second order approximation (Theorem 2.4) is accurate enough for usual values of the fixed part of dividends (that is few $\%$ of the spot value) and for maturities smaller than five years. For larger dividends or longer maturities, the third order approximation (Theorem 2.5) yields additional accuracy. Moreover, compared to other methods, these expansions are quicker to evaluate (or as quick as [BGS03]). Finally, we mention several possible extensions. Combining the stochastic expansion approaches recently developed in [BGM09, BGM10a, BGM10c, BGM10b with the current work, we could generalize the closed formulae to local or stochastic volatility models, including Gaussian stochastic interest rates. This is left to further research.

\section{A Proof of Lemma 1.1}

This is proved by induction. The result is true for $n=1$, considering (1.5). Suppose it is true for any $n(\geq 1)$ dates $\left(t_{i}\right)_{1 \leq i \leq n}$ and consider that an extra dividend payment is made at time $t_{n+1} \in\left(t_{n}, T\right]$. Then, we have

$$
\begin{aligned}
S_{T}^{(y, \delta)}= & \left(1-y_{n+1}\right) S_{t_{n+1}}^{(y, \delta)}-\frac{S_{T}}{S_{t_{n+1}}}-\delta_{n+1} \frac{S_{T}}{S_{t_{n+1}}} \\
= & \left(1-y_{n+1}\right) \frac{S_{T}}{S_{t_{n+1}}}\left[\left(\prod_{i=1}^{n}\left(1-y_{i}\right)\right) S_{t_{n+1}}-\sum_{i=1}^{n}\left(\delta_{i} \prod_{j=i+1}^{n}\left(1-y_{j}\right)\right) \frac{S_{t_{n+1}}}{S_{t_{i}}}\right] \\
& -\delta_{n+1} \frac{S_{T}}{S_{t_{n+1}}} \\
= & \left(\prod_{i=1}^{n+1}\left(1-y_{i}\right)\right) S_{T}-\sum_{i=1}^{n}\left(\delta_{i}\left(1-y_{n+1}\right) \prod_{j=i+1}^{n}\left(1-y_{j}\right)\right) \frac{S_{T}}{S_{t_{i}}}-\delta_{n+1} \frac{S_{T}}{S_{t_{n+1}}} \\
= & \left(\prod_{i=1}^{n+1}\left(1-y_{i}\right)\right) S_{T}-\sum_{i=1}^{n+1}\left(\delta_{i} \prod_{j=i+1}^{n+1}\left(1-y_{j}\right)\right) \frac{S_{T}}{S_{t_{i}}} .
\end{aligned}
$$


Thus, it is proved for $n+1$ dates.

\section{References}

[BGM09] E. Benhamou, E. Gobet, and M. Miri. Smart expansion and fast calibration for jump diffusion. Finance and Stochastics, 13(4):563-589, 2009.

[BGM10a] E. Benhamou, E. Gobet, and M. Miri. Analytical formulas for local volatility model with stochastic rates. Tentatively accepted for publication in Quantitative Finance, 2010.

[BGM10b] E. Benhamou, E. Gobet, and M. Miri. Expansion formulas for European options in a local volatility model. International Journal of Theoretical and Applied Finance, 13(4):603-634, 2010.

[BGM10c] E. Benhamou, E. Gobet, and M. Miri. Time dependent Heston model. SIAM Journal on Financial Mathematics, 1:289-325, 2010 .

[BGS03] R. Bos, A. Gairat, and D. Shepeleva. Dealing with discrete dividends. Risk Magazine, pages 109-112, 2003.

[BS88] M. Brenner and M.G. Subrahmanyam. A simple formula to compute the implied standard deviation. Financial Analysts Journal, 44:80-83, Sept./Oct. 1988.

[Bue10] H. Buehler. Volatility and dividends. Preprint TU Berlin, 2010.

[HHL03] E.G. Haug, J. Haug, and A. Lewis. Back to Basics: a New Approach to the Discrete Dividend Problem. Wilmott Magazine, pages 37-47, september 2003.

[KR05] R. Korn and L. C. G. Rogers. Stocks paying discrete dividends: modelling and option pricing. The Journal of Derivatives, 13(2):44-48, 2005.

[Nua06] D. Nualart. Malliavin calculus and related topics. Springer Verlag, second edition, 2006. 


\begin{tabular}{cccccccc}
\hline$t_{1}$ & $K$ & Monte Carlo & EG3 & VNRE & VN1000 & VW & BGS \\
& & & & & & & \\
\hline 0.1 & 70 & $24.8962\left( \pm 11.10^{-4}\right)$ & 24.8787 & 24.90 & 24.92 & 24.8862 & 24.71 \\
& 100 & $17.4338\left( \pm 12.10^{-4}\right)$ & 17.4255 & 17.43 & 17.46 & 17.4394 & 17.42 \\
& 130 & $12.3994\left( \pm 12.10^{-4}\right)$ & 12.396 & 12.40 & 12.43 & 12.4114 & 12.50 \\
& & & & & & & \\
0.5 & 70 & $26.0806\left( \pm 11.10^{-4}\right)$ & 26.0678 & 26.08 & 26.10 & 26.0752 & 25.87 \\
& 100 & $18.4815\left( \pm 12.10^{-4}\right)$ & 18.476 & 18.48 & 18.50 & 18.489 & 18.45 \\
& 130 & $13.2844\left( \pm 11.10^{-4}\right)$ & 13.283 & 13.29 & 13.31 & 13.2968 & 13.38 \\
& & & & & & & \\
0.9 & 70 & $27.2341\left( \pm 10.10^{-4}\right)$ & 27.205 & 27.21 & 27.23 & 27.2117 & 26.99 \\
& 100 & $19.4817\left( \pm 11.10^{-4}\right)$ & 19.4784 & 19.48 & 19.5 & 19.4905 & 19.43 \\
& 130 & $14.1296\left( \pm 10.10^{-4}\right)$ & 14.1293 & 14.13 & 14.16 & 14.1419 & 14.06 \\
\hline
\end{tabular}

Table 1: European Call option prices, with $\sigma=25 \%, r=6 \%, q=0 \%$, $S_{0}=100$.

[VN06] M. Vellekoop and J. Nieuwenhuis. Efficient Pricing of Derivatives on Assets with Discrete Dividends. Applied Mathematical Finance, 13(3):265-284, 2006.

[VW09] C. Veiga and U. Wystup. Closed formula for options with discrete dividends and its derivatives. Applied Mathematical Finance, 16(6):517-531, 2009. 


\begin{tabular}{|c|c|c|c|c|c|}
\hline$\sigma$ & $K$ & Monte Carlo & EG1 & EG2 & EG3 \\
\hline \multirow[t]{14}{*}{0.15} & \multirow[t]{2}{*}{40} & $59.1167\left( \pm 10^{-4}\right)$ & 59.1167 & 59.1168 & 59.1167 \\
\hline & & $15.54 \%$ & $15.54 \%$ & $15.54 \%$ & $15.54 \%$ \\
\hline & \multirow[t]{2}{*}{60} & $42.4801\left( \pm 10^{-4}\right)$ & 42.4794 & 42.4801 & 42.4801 \\
\hline & & $15.48 \%$ & $15.46 \%$ & $15.48 \%$ & $15.48 \%$ \\
\hline & \multirow[t]{2}{*}{80} & $26.8353\left( \pm 10^{-4}\right)$ & 26.8341 & 26.8353 & 26.8353 \\
\hline & & $15.43 \%$ & $15.42 \%$ & $15.43 \%$ & $15.43 \%$ \\
\hline & \multirow[t]{2}{*}{100} & $14.5117\left( \pm 10^{-4}\right)$ & 14.5106 & 14.5117 & 14.5117 \\
\hline & & $15.39 \%$ & $15.39 \%$ & $15.39 \%$ & $15.39 \%$ \\
\hline & \multirow[t]{2}{*}{120} & $6.8080\left( \pm 10^{-4}\right)$ & 6.8071 & 6.8080 & 6.8080 \\
\hline & & $15.37 \%$ & $15.36 \%$ & $15.37 \%$ & $15.37 \%$ \\
\hline & \multirow[t]{2}{*}{180} & $0.4122\left( \pm 6.10^{-5}\right)$ & 0.4118 & 0.4122 & 0.4122 \\
\hline & & $15.32 \%$ & $15.31 \%$ & $15.32 \%$ & $15.32 \%$ \\
\hline & \multirow[t]{2}{*}{250} & $0.01073\left( \pm 10^{-5}\right)$ & 0.01069 & 0.01073 & 0.01073 \\
\hline & & $15.28 \%$ & $15.28 \%$ & $15.28 \%$ & $15.28 \%$ \\
\hline \multirow[t]{14}{*}{0.25} & \multirow[t]{2}{*}{40} & $59.2155\left( \pm 10^{-4}\right)$ & 59.2139 & 59.2156 & 59.2156 \\
\hline & & $25.95 \%$ & $25.89 \%$ & $25.95 \%$ & $25.95 \%$ \\
\hline & \multirow[t]{2}{*}{60} & $43.5785\left( \pm 10^{-4}\right)$ & 43.5761 & 43.5785 & 43.5785 \\
\hline & & $25.81 \%$ & $25.79 \%$ & $25.81 \%$ & $25.81 \%$ \\
\hline & \multirow[t]{2}{*}{80} & $30.3914\left( \pm 10^{-4}\right)$ & 30.3894 & 30.3914 & 30.3914 \\
\hline & & $25.72 \%$ & $25.72 \%$ & $25.72 \%$ & $25.72 \%$ \\
\hline & \multirow[t]{2}{*}{100} & $20.3863\left( \pm 10^{-4}\right)$ & 20.3848 & 20.3863 & 20.3863 \\
\hline & & $25.66 \%$ & $25.66 \%$ & $25.66 \%$ & $25.66 \%$ \\
\hline & \multirow[t]{2}{*}{120} & $13.3435\left( \pm 10^{-4}\right)$ & 13.3418 & 13.3434 & 13.3435 \\
\hline & & $25.62 \%$ & $25.62 \%$ & $25.62 \%$ & $25.62 \%$ \\
\hline & \multirow[t]{2}{*}{180} & $3.54295\left( \pm 6.10^{-5}\right)$ & 3.5417 & 3.54294 & 3.54294 \\
\hline & & $25.54 \%$ & $25.54 \%$ & $25.54 \%$ & $25.54 \%$ \\
\hline & \multirow[t]{2}{*}{250} & $0.76946\left( \pm 4.10^{-5}\right)$ & 0.768914 & 0.769468 & 0.769468 \\
\hline & & $25.48 \%$ & $25.48 \%$ & $25.48 \%$ & $25.48 \%$ \\
\hline \multirow[t]{14}{*}{0.45} & \multirow[t]{2}{*}{40} & $61.2419\left( \pm 3.10^{-4}\right)$ & 61.2357 & 61.2418 & 61.2419 \\
\hline & & $46.79 \%$ & $46.75 \%$ & $46.79 \%$ & $46.79 \%$ \\
\hline & \multirow[t]{2}{*}{60} & $49.1493\left( \pm 10^{-4}\right)$ & 49.1447 & 49.1492 & 49.1493 \\
\hline & & $46.52 \%$ & $46.50 \%$ & $46.52 \%$ & $46.52 \%$ \\
\hline & \multirow[t]{2}{*}{80} & $39.5939\left( \pm 10^{-4}\right)$ & 39.5902 & 39.5939 & 39.5939 \\
\hline & & $46.35 \%$ & $46.35 \%$ & $46.35 \%$ & $46.35 \%$ \\
\hline & \multirow[t]{2}{*}{100} & $32.1092\left( \pm 10^{-4}\right)$ & 32.1058 & 32.1092 & 32.1092 \\
\hline & & $46.25 \%$ & $46.25 \%$ & $46.25 \%$ & $46.25 \%$ \\
\hline & \multirow[t]{2}{*}{120} & $26.2372\left( \pm 10^{-4}\right)$ & 26.2341 & 26.2372 & 26.2372 \\
\hline & & $46.17 \%$ & $46.16 \%$ & $46.17 \%$ & $46.17 \%$ \\
\hline & \multirow[t]{2}{*}{180} & $14.9691\left( \pm 10^{-4}\right)$ & 14.9665 & 14.9691 & 14.9692 \\
\hline & & $46.01 \%$ & $46.01 \%$ & $46.01 \%$ & $46.01 \%$ \\
\hline & \multirow[t]{2}{*}{250} & $8.3702\left( \pm 7.10^{-5}\right)$ & 348.3683 & 8.3702 & 8.3702 \\
\hline & & $45.91 \%$ & $45.90 \%$ & $45.91 \%$ & $45.91 \%$ \\
\hline
\end{tabular}

Table 2: European Call option prices, with $r=6 \%, q=0 \%, S_{0}=100$, $n=3, y \equiv 0.02$ and $\delta \equiv 2$. 


\begin{tabular}{|c|c|c|c|c|c|}
\hline$\delta$ & $K$ & Monte Carlo & EG1 & EG2 & EG3 \\
\hline \multirow[t]{14}{*}{2} & \multirow[t]{2}{*}{40} & $59.2155\left( \pm 10^{-4}\right)$ & 59.2139 & 59.2156 & 59.2156 \\
\hline & & $25.95 \%$ & $25.89 \%$ & $25.95 \%$ & $25.95 \%$ \\
\hline & \multirow[t]{2}{*}{60} & $43.5785\left( \pm 10^{-4}\right)$ & 43.5761 & 43.5785 & 43.5785 \\
\hline & & $25.81 \%$ & $25.80 \%$ & $25.81 \%$ & $25.81 \%$ \\
\hline & \multirow[t]{2}{*}{80} & $30.3914\left( \pm 10^{-4}\right)$ & 30.3894 & 30.3914 & 30.3914 \\
\hline & & $25.72 \%$ & $25.72 \%$ & $25.72 \%$ & $25.72 \%$ \\
\hline & \multirow[t]{2}{*}{100} & $20.3863\left( \pm 10^{-4}\right)$ & 30.3846 & 20.3863 & 20.3863 \\
\hline & & $25.66 \%$ & $25.66 \%$ & $25.66 \%$ & $25.66 \%$ \\
\hline & \multirow[t]{2}{*}{120} & $13.3435\left( \pm 10^{-4}\right)$ & 13.3418 & 13.3434 & 13.3435 \\
\hline & & $25.62 \%$ & $25.62 \%$ & $25.62 \%$ & $25.62 \%$ \\
\hline & \multirow[t]{2}{*}{180} & $3.54295\left( \pm 6.10^{-5}\right)$ & 3.5417 & 3.54294 & 3.54294 \\
\hline & & $25.54 \%$ & $25.54 \%$ & $25.54 \%$ & $25.54 \%$ \\
\hline & \multirow[t]{2}{*}{250} & $0.76946\left( \pm 5.10^{-5}\right)$ & 0.76891 & 0.76946 & 0.76946 \\
\hline & & $25.48 \%$ & $25.48 \%$ & $25.48 \%$ & $25.48 \%$ \\
\hline \multirow[t]{14}{*}{6} & \multirow[t]{2}{*}{40} & $52.3496\left( \pm 10^{-4}\right)$ & 52.3301 & 52.35 & 52.3498 \\
\hline & & $28.08 \%$ & $27.72 \%$ & $28.09 \%$ & $28.08 \%$ \\
\hline & \multirow[t]{2}{*}{60} & $37.4215\left( \pm 2.10^{-4}\right)$ & 37.3999 & 37.4209 & 37.4215 \\
\hline & & $27.60 \%$ & $27.51 \%$ & $27.59 \%$ & $27.60 \%$ \\
\hline & \multirow[t]{2}{*}{80} & $25.4276\left( \pm 2.10^{-4}\right)$ & 25.4101 & 25.4269 & 25.4276 \\
\hline & & $27.32 \%$ & $27.28 \%$ & $27.32 \%$ & $27.32 \%$ \\
\hline & \multirow[t]{2}{*}{100} & $16.7041\left( \pm 3.10^{-4}\right)$ & 16.6888 & 16.7035 & 16.704 \\
\hline & & $27.12 \%$ & $27.10 \%$ & $27.12 \%$ & $27.12 \%$ \\
\hline & \multirow[t]{2}{*}{120} & $10.7590\left( \pm 2.10^{-4}\right)$ & 10.7445 & 10.7587 & 10.759 \\
\hline & & 26. & $26.96 \%$ & $26.98 \%$ & $26.98 \%$ \\
\hline & \multirow[t]{2}{*}{180} & 2.7773 & 2.767 & 2.7774 & 2.7773 \\
\hline & & 26 & $26.69 \%$ & $26.72 \%$ & $26.72 \%$ \\
\hline & \multirow[t]{2}{*}{250} & $0.5954\left( \pm 2.10^{-4}\right)$ & 0.5912 & 0.5956 & 0.5955 \\
\hline & & $26.54 \%$ & $26.51 \%$ & $26.54 \%$ & $26.54 \%$ \\
\hline \multirow[t]{14}{*}{10} & \multirow[t]{2}{*}{40} & $45.6585\left( \pm 2.10^{-4}\right)$ & 45.5968 & 45.6577 & 45.6589 \\
\hline & & $30.54 \%$ & $29.85 \%$ & $30.53 \%$ & $30.54 \%$ \\
\hline & \multirow[t]{2}{*}{60} & $31.6875\left( \pm 3.10^{-4}\right)$ & 31.6308 & 31.684 & 31.6875 \\
\hline & & $29.64 \%$ & $29.44 \%$ & $29.63 \%$ & $29.64 \%$ \\
\hline & \multirow[t]{2}{*}{80} & $21.0058\left( \pm 3.10^{-4}\right)$ & 20.9594 & 21.0028 & 21.0058 \\
\hline & & $29.12 \%$ & $29.02 \%$ & $29.12 \%$ & $29.12 \%$ \\
\hline & \multirow[t]{2}{*}{100} & $13.5385\left( \pm 4.10^{-4}\right)$ & 13.4963 & 13.5363 & 13.5384 \\
\hline & & $28.77 \%$ & $28.69 \%$ & $28.77 \%$ & $28.77 \%$ \\
\hline & \multirow[t]{2}{*}{120} & $8.596\left( \pm 4.10^{-4}\right)$ & 8.55617 & 8.595 & 8.596 \\
\hline & & $28.52 \%$ & $28.44 \%$ & $28.52 \%$ & $28.52 \%$ \\
\hline & \multirow[t]{2}{*}{180} & $2.1656\left( \pm 2.10^{-4}\right)$ & 2.1395 & 2.1661 & 2.1656 \\
\hline & & $28.03 \%$ & $27.95 \%$ & $28.04 \%$ & $28.04 \%$ \\
\hline & \multirow[t]{2}{*}{250} & $0.4595\left( \pm 10^{-4}\right)$ & 0.4492 & 0.4601 & 0.4595 \\
\hline & & $27.71 \%$ & $27.61 \%$ & $27.72 \%$ & $27.71 \%$ \\
\hline
\end{tabular}

Table 3: European Call option prices, with $\sigma=25 \%, r=6 \%, q=0 \%$, $S_{0}=100, n=3, y \equiv 0.02$. 


\begin{tabular}{|c|c|c|c|c|c|}
\hline$n$ & $K$ & Monte Carlo & EG1 & EG2 & EG3 \\
\hline \multirow[t]{14}{*}{3} & \multirow[t]{2}{*}{40} & $55.7665\left( \pm 10^{-4}\right)$ & 55.7589 & 55.7667 & 55.7666 \\
\hline & & & $26.99 \%$ & $26.98 \%$ & $26.98 \%$ \\
\hline & \multirow[t]{2}{*}{60} & $40.453\left( \pm 10^{-4}\right)$ & 40.4433 & 40.4529 & 40.4531 \\
\hline & & $26.67 \%$ & $26.62 \%$ & $26.67 \%$ & $26.67 \%$ \\
\hline & \multirow[t]{2}{*}{80} & $27.8439\left( \pm 10^{-4}\right)$ & 27.8359 & 27.8437 & 27.8439 \\
\hline & & $26.49 \%$ & $26.47 \%$ & $26.49 \%$ & $26.49 \%$ \\
\hline & \multirow[t]{2}{*}{100} & $18.4796\left( \pm 2.10^{-4}\right)$ & 18.4727 & 18.4794 & 18.4795 \\
\hline & & $26.49 \%$ & $26.47 \%$ & $26.49 \%$ & $26.49 \%$ \\
\hline & \multirow[t]{2}{*}{120} & $11.996\left( \pm 2.10^{-4}\right)$ & 11.9896 & 11.9959 & 11.996 \\
\hline & & $26.28 \%$ & $26.27 \%$ & $26.28 \%$ & $26.28 \%$ \\
\hline & \multirow[t]{2}{*}{180} & $3.139\left( \pm 10^{-4}\right)$ & 3.1342 & 3.139 & 3.139 \\
\hline & & $26.11 \%$ & $26.10 \%$ & $26.11 \%$ & $26.11 \%$ \\
\hline & \multirow[t]{2}{*}{250} & $0.6771\left( \pm 10^{-4}\right)$ & 0.6751 & 0.6772 & 0.6771 \\
\hline & & $26.00 \%$ & $25.98 \%$ & $26.00 \%$ & $26.00 \%$ \\
\hline \multirow[t]{14}{*}{5} & \multirow[t]{2}{*}{40} & $50.4452\left( \pm 2.10^{-4}\right)$ & 50.3835 & 50.443 & 50.4454 \\
\hline & & $28.74 \%$ & $28.24 \%$ & $28.72 \%$ & $28.74 \%$ \\
\hline & \multirow[t]{2}{*}{60} & $38.3287\left( \pm 2.10^{-4}\right)$ & 38.2832 & 38.3259 & 38.3287 \\
\hline & & $28.16 \%$ & $28.02 \%$ & $28.15 \%$ & $28.16 \%$ \\
\hline & \multirow[t]{2}{*}{80} & $28.6548\left( \pm 3.10^{-4}\right)$ & 28.6226 & 28.6526 & 28.6547 \\
\hline & & $27.82 \%$ & $27.76 \%$ & $27.82 \%$ & $27.82 \%$ \\
\hline & \multirow[t]{2}{*}{100} & $21.2744\left( \pm 3.10^{-4}\right)$ & 21.2469 & 21.2727 & 21.2743 \\
\hline & & & $27.55 \%$ & $27.59 \%$ & $27.59 \%$ \\
\hline & \multirow[t]{2}{*}{120} & $15.7763\left( \pm 3.10^{-4}\right)$ & 15.7489 & 15.7751 & 15.7763 \\
\hline & & $27.42 \%$ & $27.38 \%$ & $27.42 \%$ & $27.42 \%$ \\
\hline & \multirow[t]{2}{*}{180} & $6.5681\left( \pm 3.10^{-4}\right)$ & 6.5398 & 6.5678 & 6.5681 \\
\hline & & 27 & $27.06 \%$ & $27.10 \%$ & $27.10 \%$ \\
\hline & \multirow[t]{2}{*}{250} & 2.5235 & 2.5028 & 2.5238 & 2.5236 \\
\hline & & $26.89 \%$ & $26.84 \%$ & $26.89 \%$ & $26.89 \%$ \\
\hline \multirow[t]{14}{*}{10} & \multirow[t]{2}{*}{40} & $40.8289\left( \pm 6.10^{-4}\right)$ & 40.5377 & 40.7745 & 40.8189 \\
\hline & & $34.00 \%$ & $32.85 \%$ & $33.79 \%$ & $33.96 \%$ \\
\hline & \multirow[t]{2}{*}{60} & $33.9169\left( \pm 7.10^{-4}\right)$ & 33.7164 & 33.8808 & 33.9108 \\
\hline & & & $32.03 \%$ & $32.43 \%$ & $32.50 \%$ \\
\hline & \multirow[t]{2}{*}{80} & $28.3448\left( \pm 7.10^{-4}\right)$ & 28.1848 & 28.3187 & 28.3412 \\
\hline & & $31.65 \%$ & $31.36 \%$ & $31.61 \%$ & $31.65 \%$ \\
\hline & \multirow[t]{2}{*}{100} & $23.8454\left( \pm 7.10^{-4}\right)$ & 23.6978 & 23.8247 & 23.8434 \\
\hline & & $31.08 \%$ & $30.85 \%$ & $31.05 \%$ & $31.08 \%$ \\
\hline & \multirow[t]{2}{*}{120} & $20.1926\left( \pm 8.10^{-4}\right)$ & 20.0443 & 20.1753 & 20.1916 \\
\hline & & & $30.45 \%$ & $30.64 \%$ & $30.66 \%$ \\
\hline & \multirow[t]{2}{*}{180} & $12.7129\left( \pm 7.10^{-4}\right)$ & 12.549 & 12.7027 & 12.7134 \\
\hline & & $29.88 \%$ & $29.66 \%$ & $29.86 \%$ & $29.88 \%$ \\
\hline & \multirow[t]{2}{*}{250} & $7.8484\left( \pm 7.10^{-4}\right)^{36}$ & 7.6860 & 7.8447 & 7.8495 \\
\hline & & $29.35 \%$ & $29.11 \%$ & $29.35 \%$ & $29.35 \%$ \\
\hline
\end{tabular}

Table 4: European Call option, with $\sigma=25 \%, r=6 \%, q=0 \%, S_{0}=100$, $y \equiv 0.02, \delta \equiv 4$. 


\begin{tabular}{cccc}
\hline$\delta$ & 2 & 6 & 10 \\
\hline $\mathbb{P}\left(S_{T}^{(y, \delta)}<0\right)$ & 0 & $5.10^{-10}$ & $7.10^{-7}$ \\
\hline
\end{tabular}

Table 5: $\mathbb{P}\left(S_{T}^{(y, \delta)}<0\right)$, with $\sigma=25 \%, r=6 \%, q=0 \%, S_{0}=100, n=3$ and $y \equiv 0.02$.

\begin{tabular}{cccc}
\hline$n$ & 3 & 5 & 10 \\
\hline $\mathbb{P}\left(S_{T}^{(y, \delta)}<0\right)$ & 0 & $3.10^{-6}$ & 0.023 \\
\hline
\end{tabular}

Table 6: $\mathbb{P}\left(S_{T}^{(y, \delta)}<0\right)$, with $\sigma=25 \%, r=6 \%, q=0 \%, S_{0}=100, \delta \equiv 4$ and $y \equiv 0.02$. 